Supporting Information

\title{
Enzyme Hydration Determines Resistance in
}

\section{Organic Cosolvents}

Haiyang Cui,${ }^{\dagger}$ Lingling Zhang,,$^{\dagger}$ Lobna Eltoukhy, ${ }^{\dagger}$ Qianjia Jiang, ${ }^{\dagger}$ Seval Kübra Korkunç, ${ }^{\dagger}$ KarlErich Jaeger, ${ }^{t, \S}$ Ulrich Schwaneberg ${ }^{*,+, \|}$ and Mehdi D. Davari ${ }^{*},+$

†Institute of Biotechnology, RWTH Aachen University, Worringerweg 3, Aachen 52074, Germany

Institute of Molecular Enzyme Technology, Heinrich Heine University Düsseldorf, Wilhelm Johnen Strasse, Jülich 52426, Germany

${ }^{\S}$ Institute of Bio-and Geosciences IBG 1: Biotechnology, Forschungszentrum Jülich GmbH, Wilhelm Johnen Strasse, Jülich 52426, Germany

"DWI-Leibniz Institute for Interactive Materials, Forckenbeckstraße 50, Aachen 52074, Germany

*Corresponding author: m.davari@biotec.rwth-aachen.de u.schwaneberg@biotec.rwth-aachen.de

Tel.: +49-241-80-20676; Fax: +49-241-80-22578 


\section{Tabel of Contents}

Overall and local structural change in each geometrical properties ................................... 6

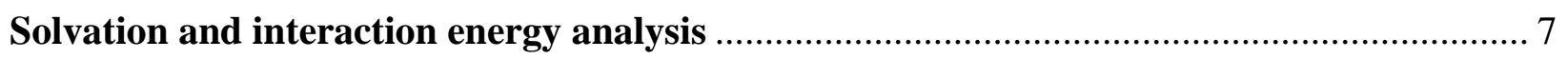

Table S1. Property of the selected ten beneficial and ten non-beneficial substitutions ................. 9

Table S2. Summary of calculated observables during MD simulation........................................ 10

Table S3. Primers used for iterative site-directed mutagenesis studies ...................................... 12

Table S4. The averaged non-bond binding energy between the overall structure of BSLA

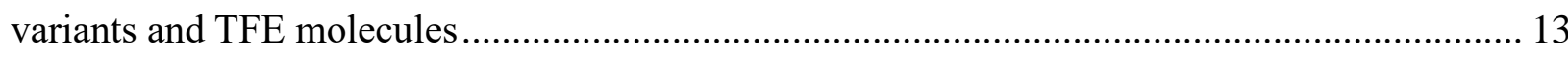

Table S5. The averaged non-bond binding energy between the overall structure of BSLA variants and water molecules.

Table S6. The averaged non-bond binding energy between the substituted residue of BSLA variants and TFE molecules

Table S7. The averaged non-bond binding energy between the substituted residue of BSLA

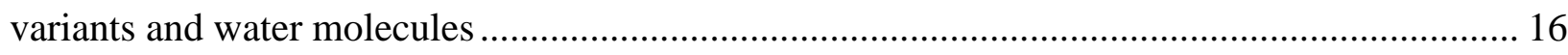

Table S8. Kinetic characterization of the purified BSLA variant in water and TFE ................... 17

Table S9. Property of the selected eight surface substitutions at four positions that exhibited decreased (increased) TFE resistance with increased (decreased) polarity.

Figure S1. Hydration shell of the beneficial and non-beneficial BSLA variants in $12 \%(\mathrm{v} / \mathrm{v})$ TFE during MD simulations .

Figure S2. TFE layer of the beneficial and non-beneficial BSLA variants in $12 \%(\mathrm{v} / \mathrm{v}) \mathrm{TFE}$ during MD simulations ....

Figure S3. The average number of TFE molecules around the BSLA substituted residue during the MD simulations in $12 \%(\mathrm{v} / \mathrm{v})$ TFE

Figure S4. Contacts frequency of substituted sites between the BSLA beneficial/non-beneficial substitution and (a) water, (b) TFE molecule in $12 \%$ (v/v) TFE, respectively.

Figure S5. The interaction energy change of BSLA beneficial and non-beneficial substitution between BSLA and water/TFE.

Figure S6. The non-bond binding energy ( $\left.\Delta \mathrm{G}_{\text {non-bond }}\right)$ change of substituted residue-water/TFE between BSLA beneficial and non-beneficial substitution on the same amino acid position...... 24 
Figure S7. Root-mean-square deviation (RMSD) of the BSLA beneficial substitutions backbone with respect to the initial structure as a function of time in $12 \%(\mathrm{v} / \mathrm{v}) \mathrm{TFE}$. 25

Figure S8. Root-mean-square deviation (RMSD) of the BSLA non-beneficial substitutions backbone with respect to the initial structure as a function of time in $12 \%(\mathrm{v} / \mathrm{v})$ TFE ............. 26 Figure S9. Time-average RMSD of BSLA substitutions determined from the last $40 \mathrm{~ns}$ of simulations in $12 \%(\mathrm{v} / \mathrm{v})$ TFE. 27

Figure S10. RMSF of each residue of BSLA beneficial substitutions determined from the last 40

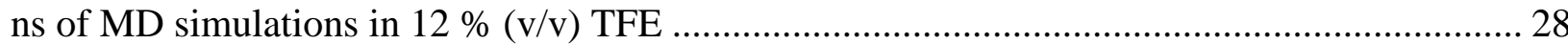

Figure S11. RMSF of each residue of BSLA non-beneficial substitutions determined from the last $40 \mathrm{~ns}$ of MD simulations in $12 \%(\mathrm{v} / \mathrm{v}) \mathrm{TFE}$

Figure S12. The time-averaged radius of gyration $\left(\mathrm{R}_{\mathrm{g}}\right)$ of BSLA beneficial and non-beneficial substitutions in $12 \%(\mathrm{v} / \mathrm{v}) \mathrm{TFE}$ 30

Figure S13. The number of internal hydrogen bonds with > 95\% occupancy in BSLA beneficial and non-beneficial substitutions 31

Figure S14. The time-averaged (a) total (b) hydrophobic (c) hydrophilic SASA of BSLA beneficial and non-beneficial substitutions 32

Figure S15. The SASA of the substituted residue of BSLA beneficial and non-beneficial substitutions in $12 \%(\mathrm{v} / \mathrm{v})$ TFE during MD simulations. 33

Figure S16. The RMSF of the substituted residue of BSLA beneficial and non-beneficial substitutions in $12 \%(\mathrm{v} / \mathrm{v})$ TFE 34

Figure S17. Averaged distance between substituted residue and the catalytic triad (a) Ser77, (b) Asp133, (c) His156 of BSLA beneficial and non-beneficial substitutions

Figure S18. The averaged internal-atomic distance between the catalytic triad (Ser77, His156, Asp133) and oxyanion hole (Ile12, Met78) of BSLA beneficial and non-beneficial substitutions

Figure S19. The average number of TFE and water molecule in substrate binding cleft of BSLA beneficial and non-beneficial substitutions during the MD simulations 37 Figure S20. $\Delta \Delta G_{\text {fold }}$ values of 20 BSLA substitutions at ten positions and grouped into three categories according to CompassR rule 38

Figure S21. Backbone root-mean-square deviation (RMSD) of the BSLA WT and recombinants with respect to the initial structure as a function of time in $12 \%(\mathrm{v} / \mathrm{v}) \mathrm{TFE}$ 39 
Figure S22. Spatial distribution of water and TFE occupancy at the surface of the BSLA variant

Figure S23. Variation of TFE resistance relative to BSLA WT as a function of TFE solvation. 41

Figure S24. Backbone root-mean-square deviation (RMSD) of the BSLA polar substitutions with respect to the initial structure as a function of time in $12 \%(\mathrm{v} / \mathrm{v})$ TFE............................ 42

Figure S25. Solvation phenomenon of BSLA polar substitutions ........................................ 43

Figure S26. RMSF of each residue of BSLA WT and recombinants determined from the last 40

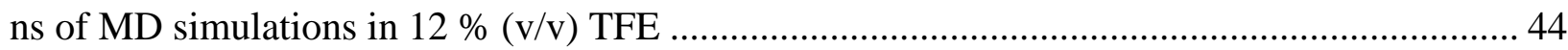

Figure S27. Time-average RMSD of BSLA WT and recombinants determined from the last 40

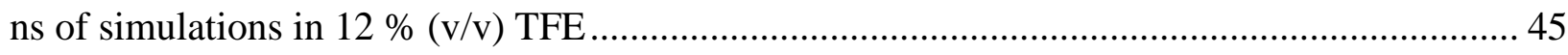

Figure S28. The time-averaged radius of gyration $\left(\mathrm{R}_{\mathrm{g}}\right)$ of BSLA WT and recombinants in $12 \%$ $(\mathrm{v} / \mathrm{v}) \mathrm{TFE}$ 46

Figure S29. The number of internal hydrogen bonds with $>95 \%$ occupancy of BSLA WT and recombinants

Figure S30. The time-averaged total, hydrophobic, hydrophilic SASA of BSLA WT and recombinants

Figure S31. The average number of TFE and water molecule in substrate binding cleft of BSLA WT and recombinants during the MD simulations..... 49

Figure S32. RMSF of each residue of BSLA WT and recombinants determined from the last 40 ns of MD simulations in $12 \%(\mathrm{v} / \mathrm{v})$ TFE . 50

Figure S33. Time-average RMSD of BSLA polar substitutions determined from the last $40 \mathrm{~ns}$ of simulations in $12 \%(\mathrm{v} / \mathrm{v})$ TFE.. 51

Figure S34. The time-averaged radius of gyration $\left(\mathrm{R}_{\mathrm{g}}\right)$ of BSLA polar substitutions in $12 \%(\mathrm{v} / \mathrm{v})$ TFE 52

Figure S35. The time-averaged total, hydrophobic, hydrophilic SASA of BSLA polar substitutions 53

Figure S36. The number of internal hydrogen bonds with $>95 \%$ occupancy of BSLA polar substitutions 54

Figure S37. The average number of TFE and water molecule in substrate binding cleft of BSLA polar substitutions during the MD simulations 55 
Figure S38. Multiple OS resistance relative to BSLA WT of the purified BSLA recombinants in

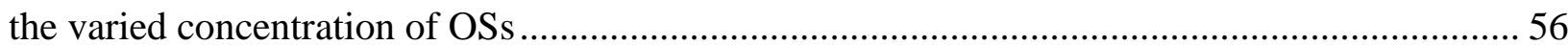




\section{Overall and local structural change in each geometrical properties}

In terms of overall conformational change, protein backbone RMSD of BSLA compared to the starting structure for simulations was calculated as a measure of BSLA stability, the time evolution of all BSLA variants are shown in Figure S7-S8. The structures of both beneficial and nonbeneficial BSLA variants were maintained in TFE throughout the $100 \mathrm{~ns}$ simulations. Fifty percent of the beneficial substitutions displayed reduced RMSD value in TFE (Figure 1b and S9), indicating that the overall structure of BSLA becomes more stabilizing comparing to nonbeneficial. While there are $20 \%$ and $30 \%$ of beneficial variants performing increased and similar RMSD value, respectively. The RMSF values for the individual residue of BSLA variants are shown in Figure S10 and S11 in SI, varying between $0.3 \AA$ and $4.0 \AA$. There was no significant flexibility change between beneficial and non-beneficial substitutions. For simulations of all substitutions, the $\mathrm{R}_{\mathrm{g}}$ is nearly $15.5 \AA$, having little difference in the first decimal place, with the fluctuations being in the same range (80\% (8/10) were unchanged, Figure 1b and S12). This denotes little variation structural compactness between beneficial and non-beneficial variant. This result can be expected because of the only one substitution introducing into the BSLA, which cannot give large variations in the overall dimensions between the different single substitutions ${ }^{1}$. Towards the number change of H-bond, decreased and similar manner of beneficial substitutions was equal (40 \% (4/10) for each, Figure 1b, and S13). Interestingly, all beneficial variants are introduced with charged amino acid into BSLA, suggesting the increased hydrophilicity ${ }^{2}$. However, $80 \%$ of beneficial substitutions had unchanged even decreased hydrophilic SASA compared to non-beneficial. Meanwhile, $50 \%$ beneficial substitutions had lower hydrophobic SASA. These "conflicted" change of SASA eventually leaded to $90 \%$ beneficial substitutions behaved similarly $(50 \%, 5 / 10)$ and lower $(40 \%, 4 / 10)$ total SASA (Figure 1b and S14). Interestingly, comparing the specific activity and resistance performance of inactive variants in TFE (Table S4), two out of four inactive variants (Y161P and N166V) resulted from the presence of TFE. As I12 is one of the residues to form the oxyanion hole, the unstructured oxyanion hole in I12P might influence the transition state of substrate, and therefore result in deactivation. In addition, the inactivation L90P might be due to the unfavorable conformational change (Figure S9, S11-14).

In terms of substituted sites, the SASA, RMSF, and the distance from the active site of substituted residue were analyzed in each BSLA substitution. $50 \%$ (5/10) of beneficial 
substitutions did not display any change compared to non-beneficial, which agrees well with the total SASA (Figure S15). The results of the RMSF of the specific substitution site are given in Figures $\mathbf{1 b}$ and S16. If one compares the flexibility of residue in beneficial and in non-beneficial, no substantial deviations are observed in $60 \%$ positons. Nevertheless, a significant variation of the flexibility occurs in the rest $40 \%$ positons, and mostly had higher flexibility (e.g., I12R, L90D, N166E), which might be caused by their long side chain in charged amino acid. This is different from the previous founding that the enzyme flexibility increased with increasing hydration level ${ }^{3}$; see hydration change description in the primary test. The distance between substitution positions and catalytic triad (S77, D133, H156) were varied from $5 \AA$ to $32 \AA$ (Figure S17). Two positons (I12, M137) were within $10 \AA$, suggesting that they might directly influence the hydrolysis reaction. Forty to eight percent of substitutions did not influence their location. Even if location change occurred, their scope of change is limited to within $3 \AA$.

In the active site, the catalytically relevant H-bond between Ser77-OG and His156-NE2 was broken because the distance significantly increased from $\sim 4.5 \AA$ (in water) ${ }^{4}$ to $6-11 \AA$ in all nonbeneficial substitutions (Figure S18a). A decreased Ser77-OG $\cdots$ His156-NE2 distance occurred in $50 \%$ of beneficial substitutions. Similar trends also happened in the oxyanion hole (Figure S18c), which might alter the stabilization state of negatively charged reaction intermediates or even result in the substrate specificity change ${ }^{4}$. In contrast, the distance between Asp133-OD1 and His156-ND1 did not show a significant change in $80 \%$ substitutions (Figure S18b), indicating that a strong H-bond (around $3.5 \AA$ ) between Asp133 and His156 are still kept in the BSLA ${ }^{4}$.

\section{Solvation and interaction energy analysis}

The phenomenon known as "water stripping" occurred to all 20 BSLA variants in TFE, as reported for enzymes in polar OSs ${ }^{4-6}$. Meanwhile, TFE interacted with the BSLA surface to form the TFE layer with inducing global disturbance in the BSLA structure (Figure S7-S18). There is no universal or predominant trend in terms of solvation in the active site (Figure S19). Furthermore, energy-based factors were calculated to examine how strong water/OS molecule binds to BSLA substitutions (Figure S5). The interaction energy includes the non-bond binding

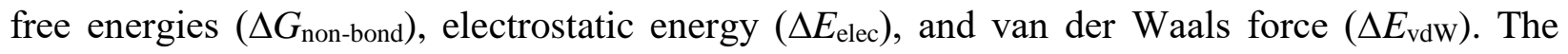
$\Delta \Delta G_{\text {non-bond }}$ value is defined as the difference between $\Delta G_{\text {non-bond, beneficial, }}$ and $\Delta G_{\text {non-bond, non-beneficial }}$ 
As shown in Figure S5, 8 out of 10 positions show superior $\Delta \Delta G_{\text {non-bond }}$ value of non-bond waters to that of non-bond TFE, especially K112P/E, its value of non-bond water reaches 5.5-fold lower than that of non-bond TFE (Figure S5). The result demonstrates that water molecules in beneficial substitutions bind to the protein more tightly than TFE molecules, despite both $\Delta \mathrm{G}_{\text {non-bond }}$ value of TFE and water on the substitution site of beneficial variants that were stronger than those in nonbeneficial variants (Figure S6). 
Table S1. Property of the selected ten beneficial and ten non-beneficial substitutions

\begin{tabular}{|c|c|c|c|c|}
\hline \multicolumn{2}{|l|}{ Substitution } & \multirow{2}{*}{$\begin{array}{l}\text { Secondary } \\
\text { structure }^{\mathrm{a}}\end{array}$} & \multirow{2}{*}{ 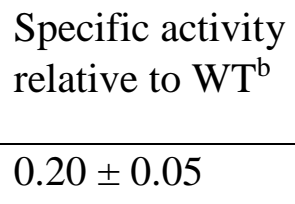 } & \multirow{2}{*}{$\begin{array}{l}\begin{array}{l}\text { Resistance } \\
\text { relative to WT }\end{array} \\
2.21 \pm 0.05\end{array}$} \\
\hline Beneficial & I12R & & & \\
\hline & G52D & $\alpha$-helix & $1.00 \pm 0.05$ & $2.83 \pm 0.12$ \\
\hline & L90D & bend & $1.01 \pm 0.05$ & $2.68 \pm 0.15$ \\
\hline & T109E & loop & $0.94 \pm 0.02$ & $1.52 \pm 0.03$ \\
\hline & $\mathrm{K} 112 \mathrm{E}$ & $\beta$-bridge & $0.69 \pm 0.04$ & $1.45 \pm 0.07$ \\
\hline & L114H & loop & $0.85 \pm 0.03$ & $1.29 \pm 0.05$ \\
\hline & M137H & loop & $1.02 \pm 0.05$ & $2.25 \pm 0.09$ \\
\hline & L140D & $\alpha$-helix & $0.78 \pm 0.03$ & $1.35 \pm 0.11$ \\
\hline & Y161D & 3/10-helix & $0.76 \pm 0.04$ & $1.28 \pm 0.06$ \\
\hline & N166E & $\alpha$-helix & $0.76 \pm 0.03$ & $2.0 \pm 0.11$ \\
\hline \multirow[t]{10}{*}{ Non-beneficial } & I12P & turn & 0 & 0 \\
\hline & G52F & $\alpha$-helix & $2.8 \pm 0.10$ & $0.20 \pm 0.05$ \\
\hline & L90P & bend & 0 & 0 \\
\hline & T109L & loop & $0.88 \pm 0.05$ & $0.10 \pm 0.07$ \\
\hline & $\mathrm{K} 112 \mathrm{P}$ & $\beta$-bridge & $0.61 \pm 0.05$ & $0.09 \pm 0.05$ \\
\hline & L114G & loop & $1.82 \pm 0.03$ & $0.05 \pm 0.07$ \\
\hline & M137I & loop & $2.60 \pm 0.05$ & $0.80 \pm 0.10$ \\
\hline & L140P & $\alpha$-helix & $0.83 \pm 0.04$ & $0.5 \pm 0.05$ \\
\hline & Y161P & 3/10-helix & $0.35 \pm 0.03$ & 0 \\
\hline & N166V & $\alpha$-helix & $0.50 \pm 0.03$ & 0 \\
\hline
\end{tabular}

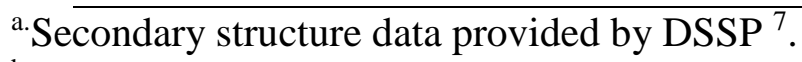

${ }^{\mathrm{b}}$ The specific activity of the purified BSLA WT in the absence/presence of $12 \%(\mathrm{v} / \mathrm{v})$ TFE was $11.5 \pm 1.2 \mathrm{U} / \mathrm{mg}$ and $4.0 \pm 0.8 \mathrm{U} / \mathrm{mg}$. One unit (U) of enzyme activity was defined as the amount of enzyme releasing $1.0 \mathrm{mmol}$ of $p$-nitrophenol per minute under the assay conditions ${ }^{8}$. 
Table S2. Summary of calculated observables during MD simulation.

\begin{tabular}{|c|c|c|c|c|}
\hline Descriptor & Location & Observables & Results & $\begin{array}{l}\text { Significant } \\
\text { determinants }\end{array}$ \\
\hline \multirow{14}{*}{$\begin{array}{l}\text { Geometrical } \\
\text { property }\end{array}$} & \multirow{6}{*}{$\begin{array}{l}\text { Overall } \\
\text { protein }\end{array}$} & Time-averaged RMSD & Fig. 1b, S7-S9 & No \\
\hline & & Time-averaged $\mathrm{R}_{\mathrm{g}}$ & Fig. 1b, S12 & No \\
\hline & & Internal H-bond & Fig. 1b, S13 & No \\
\hline & & Total SASA & Fig. 1b, S14a & No \\
\hline & & Hydrophobic SASA & Fig. 1b, S14b & No \\
\hline & & Hydrophilic SASA & Fig. 1b, S14c & No \\
\hline & \multirow{5}{*}{$\begin{array}{l}\text { Substitution } \\
\text { site }\end{array}$} & SASA of residue & Fig. 1b, S15 & No \\
\hline & & RMSF of residue & $\begin{array}{l}\text { Fig. 1b, S10-11, } \\
\text { S16 }\end{array}$ & No \\
\hline & & Distance of substitution $\cdots$ Ser77 & Fig. 1b, S17a & No \\
\hline & & Distance of substitution $\cdots$ Asp133 & Fig. 1b, S17b & No \\
\hline & & Distance of substitution $\cdots$ His 156 & Fig. 1b, S17c & No \\
\hline & \multirow[t]{3}{*}{ Active site } & $\begin{array}{l}\text { Distance of His156-ND1 } \cdots \text { Asp133- } \\
\text { OD1 }\end{array}$ & Fig. 1b, S18a & No \\
\hline & & Distance of Ser77-OG $\cdots$ His156-OD2 & Fig. 1b, S18b & No \\
\hline & & Distance of Ile12-N $\cdots$ Met78-N & Fig. 1b, S18c & No \\
\hline \multirow{8}{*}{$\begin{array}{l}\text { Solvation } \\
\text { phenomenon }\end{array}$} & \multirow{2}{*}{$\begin{array}{l}\text { Overall } \\
\text { protein }\end{array}$} & OS layer & Fig. 1b, S2 & No \\
\hline & & Hydration shell & Fig. 1b, S1 & Yes \\
\hline & \multirow{4}{*}{$\begin{array}{l}\text { Substitution } \\
\text { site }\end{array}$} & Number of TFE molecule & Fig. 1b, S3 & No \\
\hline & & Number of Water molecule & Fig. 1b, 1c & Yes \\
\hline & & OS-residue contact frequency & Fig. 1b, 1e, S4b & No \\
\hline & & Water-residue contact frequency & Fig. 1b, 1d, S4a & Yes \\
\hline & \multirow[t]{2}{*}{ Active site } & Number of TFE molecule & Fig. 1b, S19a & No \\
\hline & & Number of Water molecule & Fig. 1b, S19b & No \\
\hline \multirow{7}{*}{$\begin{array}{l}\text { Interaction } \\
\text { energy }\end{array}$} & \multirow{7}{*}{$\begin{array}{l}\text { Overall } \\
\text { protein }\end{array}$} & $\Delta \mathrm{E}_{\mathrm{vdw}}(\mathrm{BSLA}-\mathrm{TFE})$ & Fig. S5, Tab. S4 & No \\
\hline & & $\Delta \mathrm{E}_{\text {elec }}(\mathrm{BSLA}-\mathrm{TFE})$ & Fig. S5, Tab. S4 & No \\
\hline & & $\Delta \mathrm{G}_{\text {non-bond }}(\mathrm{BSLA}-\mathrm{TFE})$ & Fig. S5, Tab. S4 & No \\
\hline & & $\Delta \mathrm{E}_{\mathrm{vdW}}(\mathrm{BSLA}-\mathrm{Water})$ & Fig. S5, Tab. S5 & Yes \\
\hline & & $\Delta \mathrm{E}_{\text {elec }}(\mathrm{BSLA}-\mathrm{Water})$ & Fig. S5, Tab. S5 & Yes \\
\hline & & $\Delta \mathrm{G}_{\text {non-bond }}(\mathrm{BSLA}-$ Water) & Fig. S5, Tab. S5 & Yes \\
\hline & & $\Delta \mathrm{E}_{\mathrm{vdW}}($ Residue-TFE) & Fig. S5, Tab. S6 & No \\
\hline
\end{tabular}




\begin{tabular}{|c|c|c|c|c|}
\hline Descriptor & Location & Observables & Results & $\begin{array}{l}\text { Significant } \\
\text { determinants }\end{array}$ \\
\hline & \multirow{6}{*}{$\begin{array}{l}\text { Substitution } \\
\text { site }\end{array}$} & $\Delta \mathrm{E}_{\text {elec }}$ (Residue-TFE) & Fig. S5, Tab. S6 & No \\
\hline & & $\Delta \mathrm{G}_{\text {non-bond }}($ Residue-TFE) & Fig. S5, Tab. S6 & No \\
\hline & & $\Delta \mathrm{E}_{\mathrm{vdW}}$ (Residue-Water) & Fig. S5, Tab. S7 & Yes \\
\hline & & $\Delta \mathrm{E}_{\text {elec }}($ Residue-Water $)$ & Fig. S5, Tab. S7 & Yes \\
\hline & & $\Delta \mathrm{G}_{\text {non-bond }}$ (Residue-Water) & Fig. S5, Tab. S7 & Yes \\
\hline & & $\Delta \Delta \mathrm{G}_{\text {non-bond }}$ & Fig. S6 & Yes \\
\hline In total & & 35 & & \\
\hline
\end{tabular}


Table S3. Primers used for iterative site-directed mutagenesis studies.

\begin{tabular}{ll}
\hline Name & 5 --> 3 sequence $^{\mathrm{a}}$ \\
\hline I12R Forward Primer & ATGGTTCACGGTCGGGGAGGGG \\
I12R Reverse Primer & ATGCCCCTCCCCGACCGTGAAC \\
M137H Forward Primer & ATGATTGTCCACAATTACTTAT \\
M137H Reverse Primer & TTGATAAGTAATTGTGGACAAT \\
N166E Forward Primer & AGCAGCCAAGTCGAGAGCCTGA \\
N166E Reverse Primer & TAATCAGGCTCTCGACTTGGCT \\
K112E Forward Primer & TTGACGACAGGCGAGGCGCTT \\
K112E Reverse Primer & AAGCGCCTCGCCTGTCGTCAA
\end{tabular}

${ }^{a}$ Mutated codon is underlined. 
Table S4. The averaged non-bond binding energy between the overall structure of BSLA variants and TFE molecules. ${ }^{\text {a }}$

\begin{tabular}{|c|c|c|c|c|c|c|}
\hline \multirow{2}{*}{$\begin{array}{l}\text { Amino acid } \\
\text { Position }\end{array}$} & \multicolumn{3}{|c|}{ Non-beneficial (kcal/mol) } & \multicolumn{3}{|c|}{ Beneficial (kcal/mol) } \\
\hline & $\Delta \mathrm{E}_{\mathrm{vdW}}$ & $\Delta \mathrm{E}_{\text {elec }}$ & $\Delta \mathrm{G}_{\text {non-bond }}$ & $\Delta \mathrm{E}_{\mathrm{vdW}}$ & $\Delta \mathrm{E}_{\text {elec }}$ & $\Delta \mathrm{G}_{\text {non-bond }}$ \\
\hline 12 & $-1543 \pm 89$ & $-1721 \pm 203$ & $-3264 \pm 291$ & $-1544 \pm 88$ & $-1721 \pm 209$ & $-3265 \pm 296$ \\
\hline 52 & $-1549 \pm 94$ & $-1714 \pm 206$ & $-3263 \pm 300$ & $-1573 \pm 101$ & $-1823 \pm 226$ & $-3396 \pm 327$ \\
\hline 90 & $-1594 \pm 85$ & $-1651 \pm 182$ & $-3246 \pm 267$ & $-1527 \pm 80$ & $-1726 \pm 216$ & $-3253 \pm 295$ \\
\hline 109 & $-1491 \pm 76$ & $-1659 \pm 196$ & $-3151 \pm 272$ & $-1546 \pm 85$ & $-1708 \pm 200$ & $-3254 \pm 285$ \\
\hline 112 & $-1553 \pm 86$ & $-1755 \pm 199$ & $-3308 \pm 285$ & $-1479 \pm 101$ & $-1594 \pm 195$ & $-3073 \pm 296$ \\
\hline 114 & $-1576 \pm 92$ & $-1657 \pm 208$ & $-3233 \pm 299$ & $-1499 \pm 87$ & $-1678 \pm 234$ & $-3177 \pm 321$ \\
\hline 137 & $-1585 \pm 84$ & $-1743 \pm 194$ & $-3328 \pm 278$ & $-1528 \pm 84$ & $-1679 \pm 190$ & $-3207 \pm 275$ \\
\hline 140 & $-1643 \pm 91$ & $-1684 \pm 177$ & $-3327 \pm 267$ & $-1482 \pm 98$ & $-1850 \pm 214$ & $-3332 \pm 312$ \\
\hline 161 & $-1675 \pm 86$ & $-1870 \pm 218$ & $-3545 \pm 304$ & $-1511 \pm 83$ & $-1853 \pm 205$ & $-3364 \pm 288$ \\
\hline 166 & $-1557 \pm 83$ & $-1706 \pm 189$ & $-3263 \pm 271$ & $-1601 \pm 91$ & $-1873 \pm 206$ & $-3474 \pm 297$ \\
\hline
\end{tabular}

$\overline{{ }^{a}}$ The data were averaged from the last $40 \mathrm{~ns}$ in three independent $\mathrm{MD}$ runs. $\Delta \mathrm{E}_{\text {elec }}$ : electrostatic energy. $\Delta \mathrm{E}_{\mathrm{vdw}}$ : van der Waals. $\Delta \mathrm{G}_{\mathrm{non}}$ bond: the non-bond binding. $\Delta \mathrm{G}_{\text {non-bond }}=\Delta \mathrm{E}_{\mathrm{elec}}+\Delta \mathrm{E}_{\mathrm{vdW}}$. 
Table S5. The averaged non-bond binding energy between the overall structure of BSLA variants and water molecules. ${ }^{\mathrm{a}}$

\begin{tabular}{|c|c|c|c|c|c|c|}
\hline \multirow{2}{*}{$\begin{array}{l}\text { Amino acid } \\
\text { Position }\end{array}$} & \multicolumn{3}{|c|}{ Non-beneficial (kcal/mol) } & \multicolumn{3}{|c|}{ Beneficial (kcal/mol) } \\
\hline & $\Delta \mathrm{E}_{\mathrm{vdW}}$ & $\Delta \mathrm{E}_{\text {elec }}$ & $\Delta \mathrm{G}_{\text {non-bond }}$ & $\Delta \mathrm{E}_{\mathrm{vdW}}$ & $\Delta \mathrm{E}_{\text {elec }}$ & $\Delta \mathrm{G}_{\text {non-bond }}$ \\
\hline 12 & $-8018 \pm 347$ & $40 \pm 105$ & $-7977 \pm 452$ & $-8013 \pm 351$ & $61 \pm 96$ & $-7952 \pm 447$ \\
\hline 52 & $-8203 \pm 346$ & $28 \pm 93$ & $-8174 \pm 439$ & $-8016 \pm 347$ & $48 \pm 90$ & $-7968 \pm 437$ \\
\hline 90 & $-7772 \pm 329$ & $50 \pm 89$ & $-7721 \pm 419$ & $-8456 \pm 342$ & $91 \pm 100$ & $-8365 \pm 442$ \\
\hline 109 & $-7964 \pm 346$ & $70 \pm 91$ & $-7893 \pm 439$ & $-8259 \pm 389$ & $74 \pm 101$ & $-8184 \pm 491$ \\
\hline 112 & $-7769 \pm 358$ & $14 \pm 90$ & $-7754 \pm 449$ & $-8126 \pm 341$ & $31 \pm 93$ & $-8095 \pm 435$ \\
\hline 114 & $-8221 \pm 322$ & $78 \pm 97$ & $-8142 \pm 420$ & $-8035 \pm 388$ & $38 \pm 90$ & $-7996 \pm 478$ \\
\hline 137 & $-8137 \pm 340$ & $43 \pm 92$ & $-8093 \pm 422$ & $-7939 \pm 359$ & $47 \pm 92$ & $-7891 \pm 452$ \\
\hline 140 & $-7945 \pm 347$ & $48 \pm 96$ & $-7896 \pm 443$ & $-8103 \pm 396$ & $57 \pm 93$ & $-8045 \pm 489$ \\
\hline 161 & $-8264 \pm 346$ & $52 \pm 94$ & $-8211 \pm 441$ & $-7972 \pm 318$ & $68 \pm 95$ & $-7904 \pm 413$ \\
\hline 166 & $-8103 \pm 342$ & $58 \pm 91$ & $-8044 \pm 420$ & $-8400 \pm 388$ & $48 \pm 102$ & $-8351 \pm 491$ \\
\hline
\end{tabular}

${ }^{\mathrm{a}}$ The data were averaged from the last $40 \mathrm{~ns}$ in three independent $\mathrm{MD}$ runs. $\Delta \mathrm{E}_{\text {elec}}$ : electrostatic energy. $\Delta \mathrm{E}_{\mathrm{vdw}}$ : van der $\mathrm{Waals}$. $\Delta \mathrm{G}_{\mathrm{non}}$ bond: the non-bond binding. $\Delta \mathrm{G}_{\text {non-bond }}=\Delta \mathrm{E}_{\mathrm{elec}}+\Delta \mathrm{E}_{\mathrm{vdW}}$. 
Table S6. The averaged non-bond binding energy between the substituted residue of BSLA variants and TFE molecules. ${ }^{\mathrm{a}}$

\begin{tabular}{|c|c|c|c|c|c|c|}
\hline \multirow{2}{*}{$\begin{array}{l}\text { Amino acid } \\
\text { Position }\end{array}$} & \multicolumn{3}{|c|}{ Non-beneficial (kcal/mol) } & \multicolumn{3}{|c|}{ Beneficial (kcal/mol) } \\
\hline & $\Delta \mathrm{E}_{\mathrm{vdW}}$ & $\Delta \mathrm{E}_{\text {elec }}$ & $\Delta \mathrm{G}_{\text {non-bond }}$ & $\Delta \mathrm{E}_{\mathrm{vdW}}$ & $\Delta \mathrm{E}_{\text {elec }}$ & $\Delta \mathrm{G}_{\text {non-bond }}$ \\
\hline 12 & $-23 \pm 5$ & $-3 \pm 5$ & $-26 \pm 10$ & $-27 \pm 8$ & $-39 \pm 24$ & $-66 \pm 32$ \\
\hline 52 & $-21 \pm 5$ & $-2 \pm 3$ & $-23 \pm 7$ & $-1 \pm 11$ & $-144 \pm 54$ & $-145 \pm 65$ \\
\hline 90 & $-16 \pm 5$ & $-4 \pm 8$ & $-21 \pm 13$ & $-1 \pm 8$ & $-72 \pm 43$ & $-72 \pm 52$ \\
\hline 109 & $-20 \pm 7$ & $-6 \pm 10$ & $-26 \pm 16$ & $-7 \pm 10$ & $-90 \pm 55$ & $-97 \pm 65$ \\
\hline 112 & $-15 \pm 6$ & $-4 \pm 7$ & $-19 \pm 13$ & $-4 \pm 10$ & $-60 \pm 50$ & $-64 \pm 60$ \\
\hline 114 & $-7 \pm 6$ & $-4 \pm 7$ & $-11 \pm 10$ & $-10 \pm 8$ & $-17 \pm 19$ & $-27 \pm 27$ \\
\hline 137 & $-18 \pm 7$ & $-3 \pm 5$ & $-20 \pm 12$ & $-19 \pm 8$ & $-19 \pm 18$ & $-38 \pm 26$ \\
\hline 140 & $-15 \pm 7$ & 0 & $-15 \pm 4$ & $4 \pm 9$ & $-106 \pm 59$ & $-101 \pm 68$ \\
\hline 161 & $-20 \pm 6$ & $-4 \pm 9$ & $-24 \pm 16$ & $-3 \pm 11$ & $-138 \pm 71$ & -140 v 82 \\
\hline 166 & $-7 \pm 3$ & $-1 \pm 2$ & $-8 \pm 5$ & $4 \pm 12$ & $-108 \pm 53$ & $-103 \pm 65$ \\
\hline
\end{tabular}

a The data were averaged from the last $40 \mathrm{~ns}$ in three independent $\mathrm{MD}$ runs. $\Delta \mathrm{E}_{\text {elec}}$ : electrostatic energy. $\Delta \mathrm{E}_{\mathrm{vdw}}$ : van der Waals. $\Delta \mathrm{G}_{\mathrm{non}}$ bond: the non-bond binding. $\Delta \mathrm{G}_{\text {non-bond }}=\Delta \mathrm{E}_{\mathrm{elec}}+\Delta \mathrm{E}_{\mathrm{vdW}}$. 
Table S7. The averaged non-bond binding energy between the substituted residue of BSLA variants and water molecules. ${ }^{\mathrm{a}}$

\begin{tabular}{|c|c|c|c|c|c|c|}
\hline \multirow{2}{*}{$\begin{array}{l}\text { Amino acid } \\
\text { Position }\end{array}$} & \multicolumn{3}{|c|}{ Non-beneficial (kcal/mol) } & \multicolumn{3}{|c|}{ Beneficial (kcal/mol) } \\
\hline & $\Delta \mathrm{E}_{\mathrm{vdW}}$ & $\Delta \mathrm{E}_{\text {elec }}$ & $\Delta \mathrm{G}_{\text {non-bond }}$ & $\Delta \mathrm{E}_{\mathrm{vdW}}$ & $\Delta \mathrm{E}_{\text {elec }}$ & $\Delta \mathrm{G}_{\text {non-bond }}$ \\
\hline 12 & $-2 \pm 2$ & $-3 \pm 7$ & $-5 \pm 9$ & $1 \pm 8$ & $-72 \pm 27$ & $-71 \pm 35$ \\
\hline 52 & $-2 \pm 2$ & 0 & $-3 \pm 4$ & $5 \pm 9$ & $-58 \pm 38$ & $-53 \pm 47$ \\
\hline 90 & $-6 \pm 5$ & $-12 \pm 12$ & $-18 \pm 16$ & $9 \pm 12$ & $-179 \pm 52$ & $-170 \pm 64$ \\
\hline 109 & $-4 \pm 5$ & $-27 \pm 14$ & $-31 \pm 19$ & $13 \pm 13$ & $-235 \pm 64$ & $-222 \pm 77$ \\
\hline 112 & $-9 \pm 6$ & $-13 \pm 11$ & $-23 \pm 16$ & $15 \pm 12$ & $-253 \pm 57$ & $-238 \pm 70$ \\
\hline 114 & $-1 \pm 3$ & $-7 \pm 8$ & $-9 \pm 11$ & $-3 \pm 7$ & $-59 \pm 22$ & $-62 \pm 30$ \\
\hline 137 & $-7 \pm 4$ & $-2 \pm 5$ & $-9 \pm 9$ & $-6 \pm 8$ & $-40 \pm 21$ & $-46 \pm 28$ \\
\hline 140 & $-1 \pm 1$ & 0 & $-1 \pm 1$ & $8 \pm 10$ & $-111 \pm 61$ & $-103 \pm 71$ \\
\hline 161 & $-3 \pm 5$ & $-16 \pm 12$ & $-19 \pm 18$ & $8 \pm 11$ & $-139 \pm 73$ & $-131 \pm 84$ \\
\hline 166 & $-2 \pm 1$ & 0 & $-1 \pm 2$ & $7 \pm 9$ & $-108 \pm 64$ & $-101 \pm 73$ \\
\hline
\end{tabular}

$\overline{{ }^{a}}$ The data were averaged from the last $40 \mathrm{~ns}$ in three independent $\mathrm{MD}$ runs. $\Delta \mathrm{E}_{\text {elec}}$ : electrostatic energy. $\Delta \mathrm{E}_{\mathrm{vdW}}$ : van der $\mathrm{Waals}$. $\Delta \mathrm{G}_{\mathrm{non}}-$ bond: the non-bond binding. $\Delta \mathrm{G}_{\text {non-bond }}=\Delta \mathrm{E}_{\mathrm{elec}}+\Delta \mathrm{E}_{\mathrm{vdW}}$. 
Table S8. Kinetic characterization of the purified BSLA variant in water and TFE. ${ }^{\text {a }}$

\begin{tabular}{|c|c|c|c|c|c|c|}
\hline \multirow{2}{*}{ BSLA variant } & \multicolumn{2}{|c|}{$K_{M}(\mathrm{mM})$} & \multicolumn{2}{|c|}{$k_{c \text { at }}\left(\min ^{-1}\right)$} & \multicolumn{2}{|c|}{$k_{c \mathrm{cat}} / K_{M}\left(\mathrm{mM}^{-1} \mathrm{~min}^{-1}\right)$} \\
\hline & $0 \%$ & $12 \%$ & $0 \%$ & $12 \%$ & $0 \%$ & $12 \%$ \\
\hline $\mathrm{WT}^{4}$ & 0.51 & 0.78 & 185 & 136 & 362 & 174 \\
\hline I12R & 0.53 & 0.31 & 113 & 124 & 213 & 405 \\
\hline I12R/M137H & 0.29 & 0.29 & 126 & 149 & 436 & 520 \\
\hline I12R/M137H/N166E & 0.53 & 0.45 & 65 & 80 & 123 & 179 \\
\hline I12R/M137H/N166E/K112E & 0.50 & 0.40 & 202 & 195 & 405 & 484 \\
\hline
\end{tabular}


Table S9. Property of the selected eight surface substitutions at four positions that exhibited decreased/increased TFE resistance with increased/decreased polarity.

\begin{tabular}{llll}
\hline Substitution & $\begin{array}{l}\text { Secondary } \\
\text { structure }^{\mathrm{a}}\end{array}$ & $\begin{array}{l}\text { Specific activity } \\
\text { relative to WT }\end{array}$ & $\begin{array}{l}\text { Resistance } \\
\text { relative to WT }\end{array}$ \\
\hline $\mathrm{I} 12 \mathrm{Q}$ & turn & $0.11 \pm 0.03$ & $1.69 \pm 0.07$ \\
$\mathrm{I} 12 \mathrm{~N}$ & turn & $0.10 \pm 0.03$ & $1.08 \pm 0.04$ \\
$\mathrm{~K} 112 \mathrm{~S}$ & $\beta$-bridge & $1.00 \pm 0.05$ & $0.90 \pm 0.04$ \\
$\mathrm{~K} 112 \mathrm{~T}$ & $\beta$-bridge & $1.22 \pm 0.07$ & $0.85 \pm 0.04$ \\
$\mathrm{M} 137 \mathrm{Y}$ & loop & $1.46 \pm 0.06$ & $1.70 \pm 0.07$ \\
$\mathrm{M} 137 \mathrm{~S}$ & loop & $1.07 \pm 0.04$ & $1.11 \pm 0.04$ \\
$\mathrm{~N} 166 \mathrm{Y}$ & $\alpha$-helix & $0.81 \pm 0.03$ & $1.20 \pm 0.05$ \\
$\mathrm{~N} 166 \mathrm{C}$ & $\alpha$-helix & $0.44 \pm 0.02$ & 0
\end{tabular}

a. Secondary structure data provided by DSSP ${ }^{7}$. 


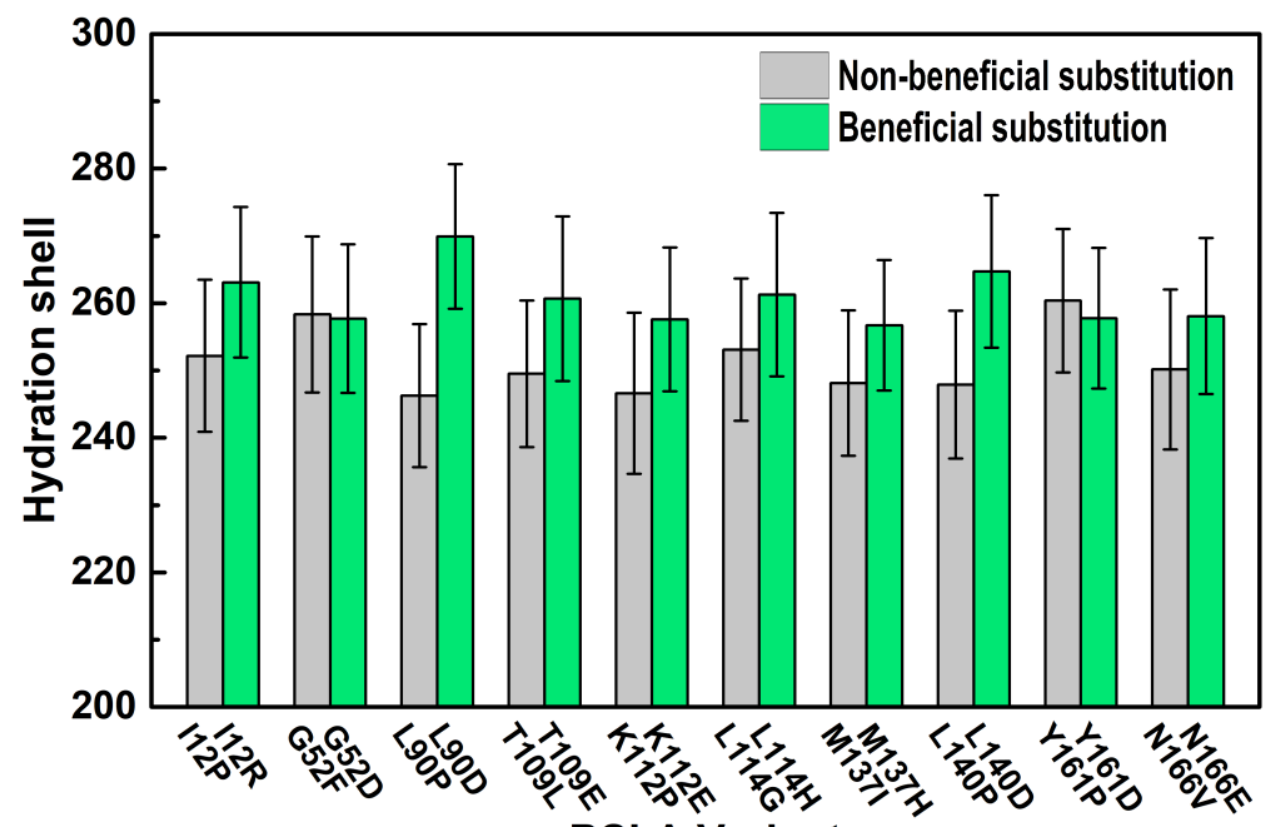

BSLA Variant

Figure S1. Hydration shell of the beneficial and non-beneficial BSLA variants in $12 \%$ (v/v) TFE during MD simulations. Water molecules whose $\mathrm{O}$ atom is within $3.5 \AA$ distance cut-off of any non-hydrogen atom of BSLA were described as the first hydration shell. The data of the TFE layer was averaged from three independent simulation runs. 


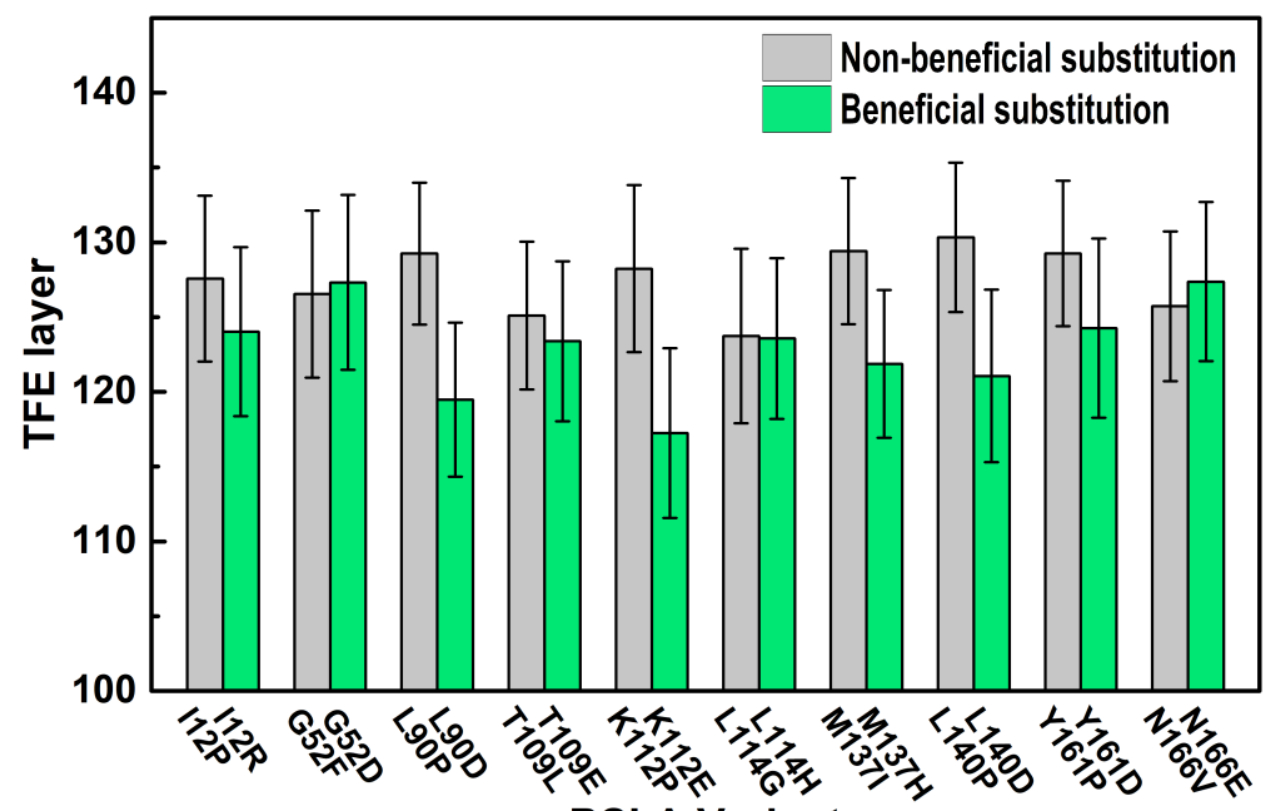

BSLA Variant

Figure S2. TFE layer of the beneficial and non-beneficial BSLA variants in $12 \%(\mathrm{v} / \mathrm{v})$ TFE during MD simulations. A similar definition with the hydrogen level is also defined for the organic solvent layer. A $6.8 \AA$ cut-off was employed for TFE as a previous study ${ }^{4}$. The data of the TFE layer was averaged from three independent simulation runs. 


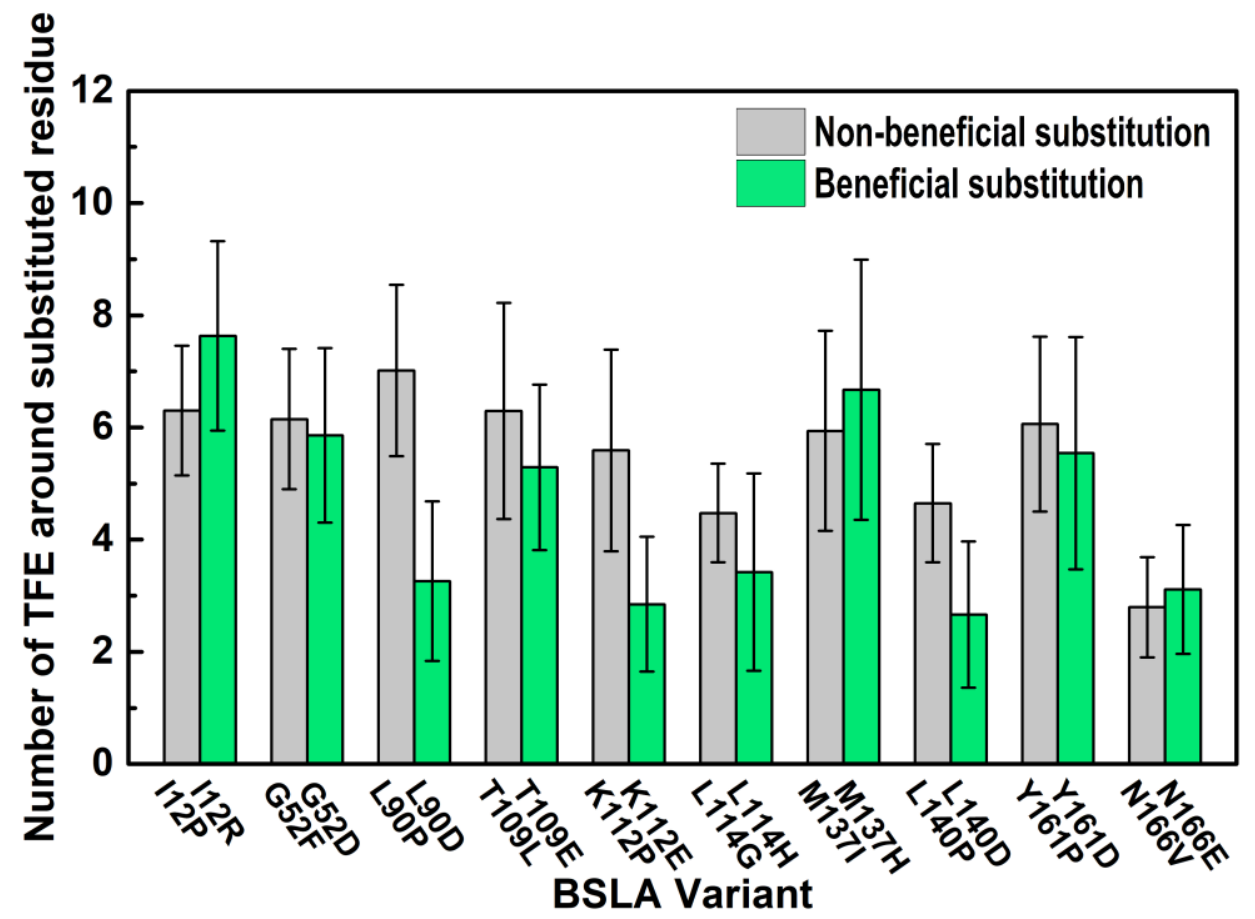

Figure S3. The average number of TFE molecules around the BSLA substituted residue during the MD simulations in $12 \%(\mathrm{v} / \mathrm{v})$ TFE. The number of TFE molecules was averaged over the last $40 \mathrm{~ns}$ from three independent MD runs. 
(a)
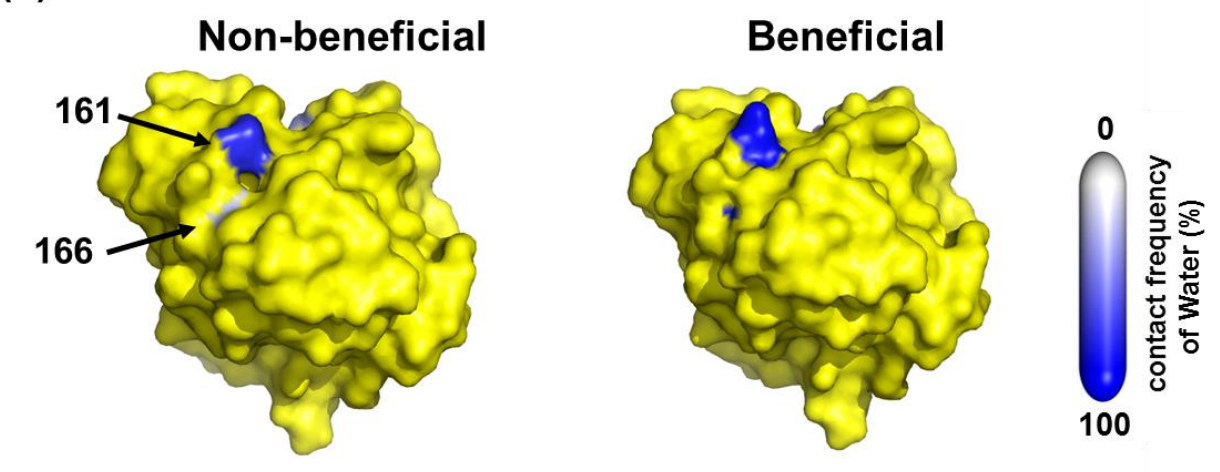

(b)

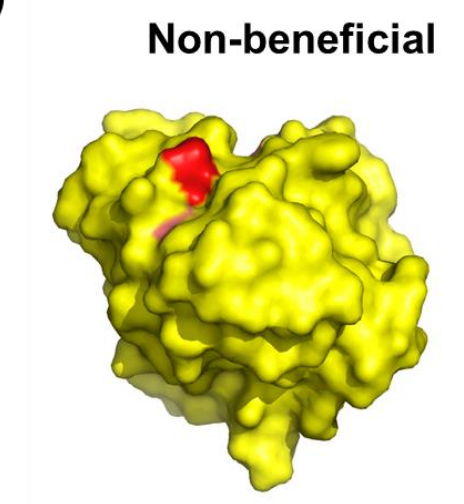

Figure S4. Contacts frequency of substituted sites between the BSLA beneficial/non-beneficial substitution and (a) water, (b) TFE molecule in $12 \%$ (v/v) TFE, respectively. Residue-water/TFE contact was defined as residue-water/TFE molecule distance being $2.5 \AA$ or less ${ }^{4}$. Contact frequency was calculated over the last $40 \mathrm{~ns}$ from three independent MD runs. The ramp was colored from white to blue/red to indicate the change of residue-water/TFE contact frequency from low to high. Specific substituted sites are labeled with amino acid position number. Except for the substituted sites, the rest surface is colored with yellow. Front-side (rotated by $180^{\circ}$ ) views are shown in Figure 2 to give a complete view of the BSLA substitutions. Each view of BSLA has the same orientation. 


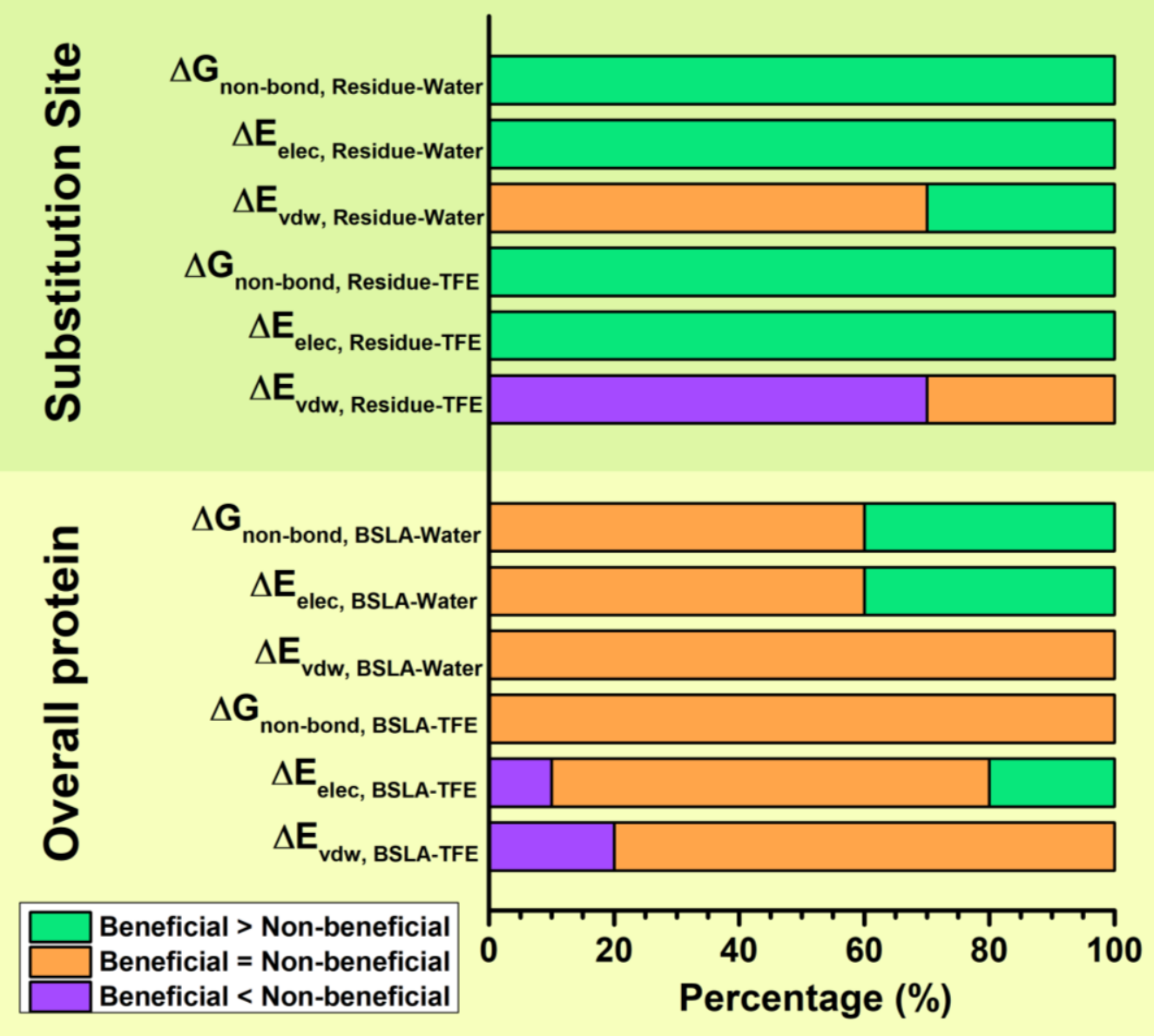

Figure S5. The interaction energy change of BSLA beneficial and non-beneficial substitution

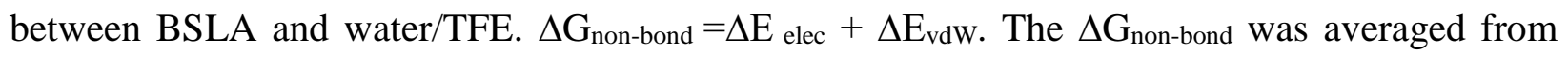
three independent simulations. The van der Waals $\left(\Delta \mathrm{E}_{\mathrm{vdW}}\right)$, and electrostatic energy $\left(\Delta \mathrm{E}_{\text {elec }}\right)$ between the substrate binding cleft residues and OS molecules were also calculated. 


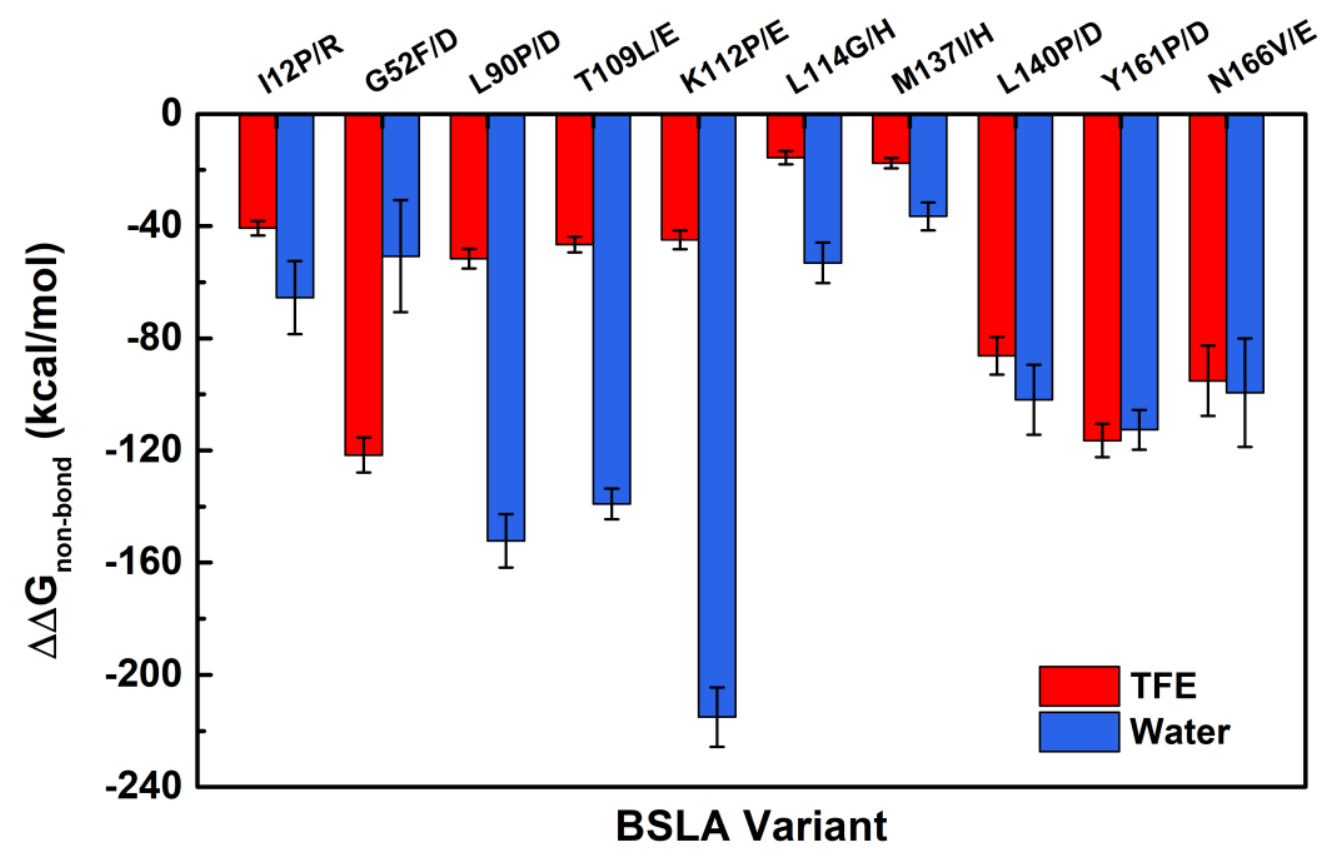

Figure S6. The non-bond binding energy ( $\left.\Delta \mathrm{G}_{\text {non-bond }}\right)$ change of substituted residue-water/TFE between BSLA beneficial and non-beneficial substitution on the same amino acid position,

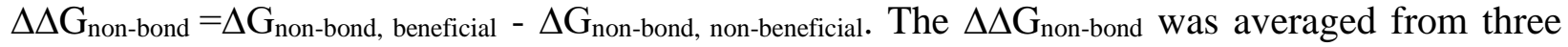
independent simulations. 


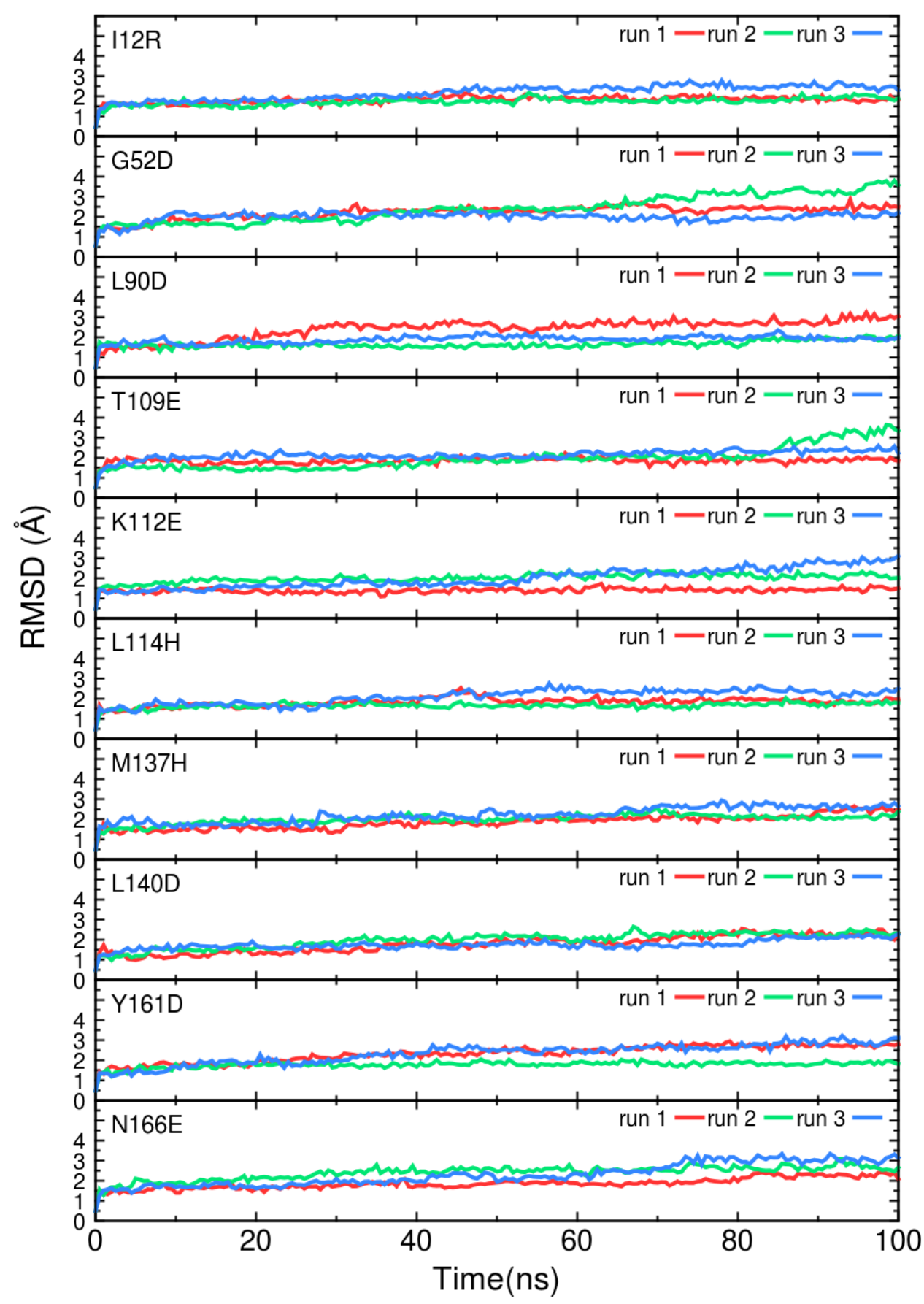

Figure S7. Root-mean-square deviation (RMSD) of the BSLA beneficial substitutions backbone with respect to the initial structure as a function of time in $12 \%(\mathrm{v} / \mathrm{v})$ TFE. Three independent MD runs for each solvent are shown. 


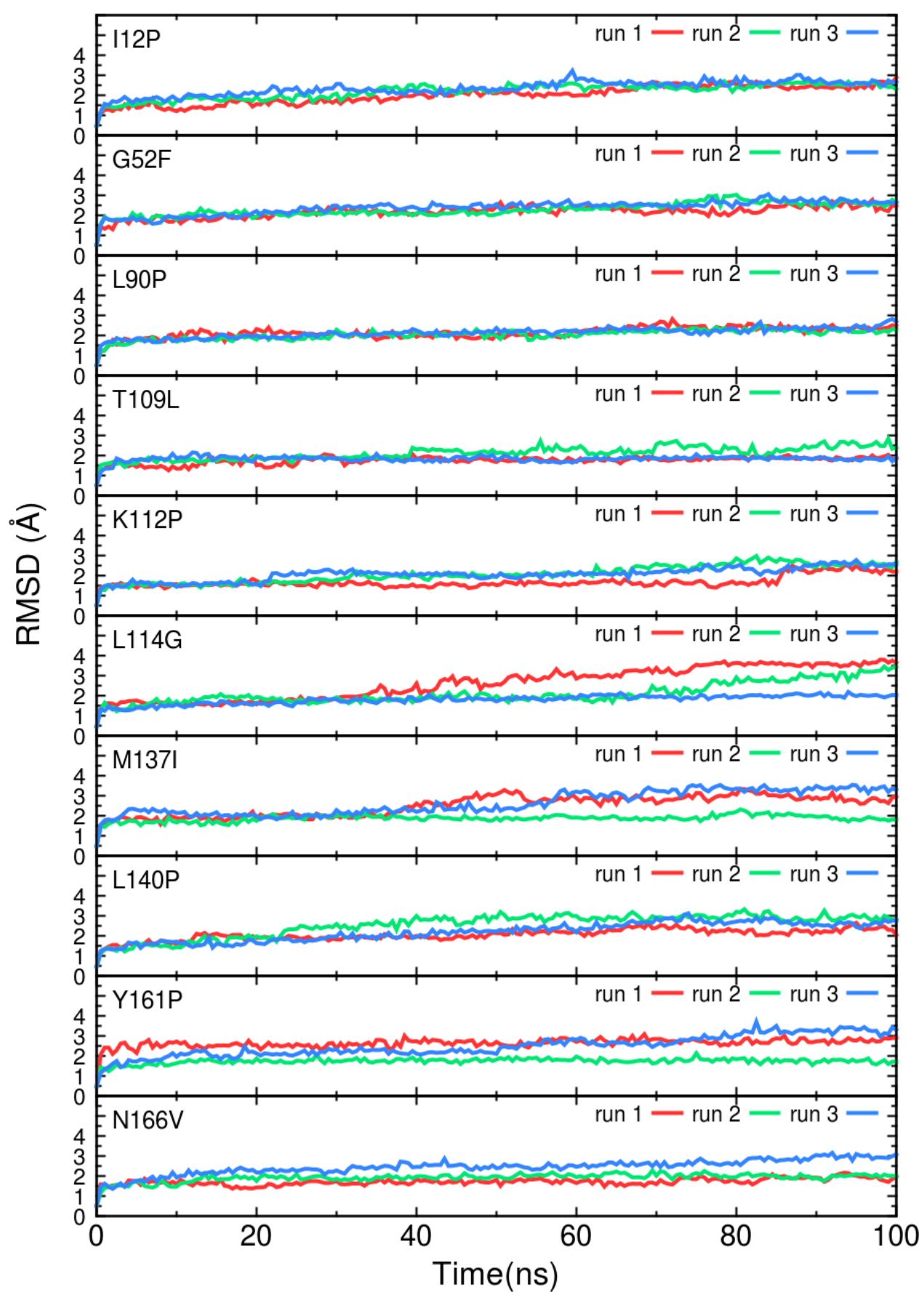

Figure S8. Root-mean-square deviation (RMSD) of the BSLA non-beneficial substitutions backbone with respect to the initial structure as a function of time in $12 \%(\mathrm{v} / \mathrm{v})$ TFE. Three independent MD runs for each solvent are shown. 


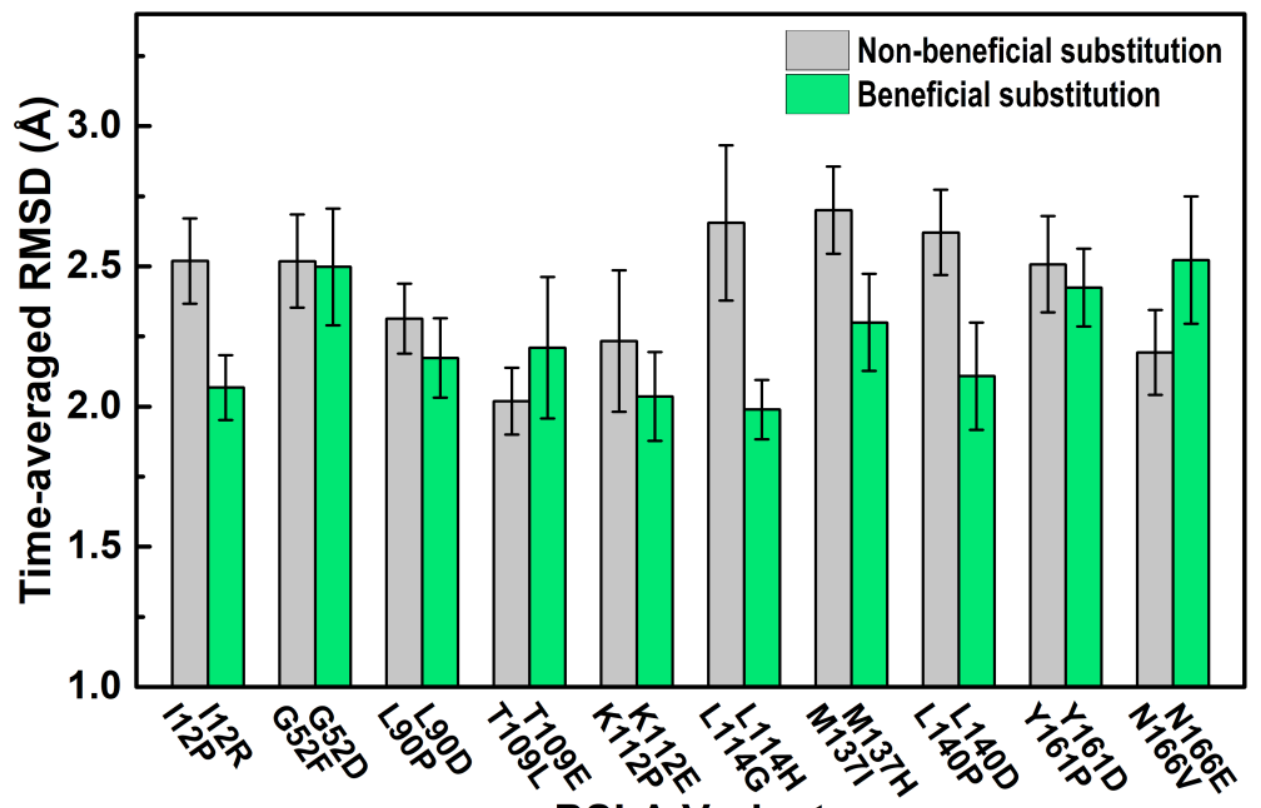

BSLA Variant

Figure S9. Time-average RMSD of BSLA substitutions determined from the last $40 \mathrm{~ns}$ of simulations in $12 \%$ (v/v) TFE. Error bars describe the standard deviation from three independent MD runs. 


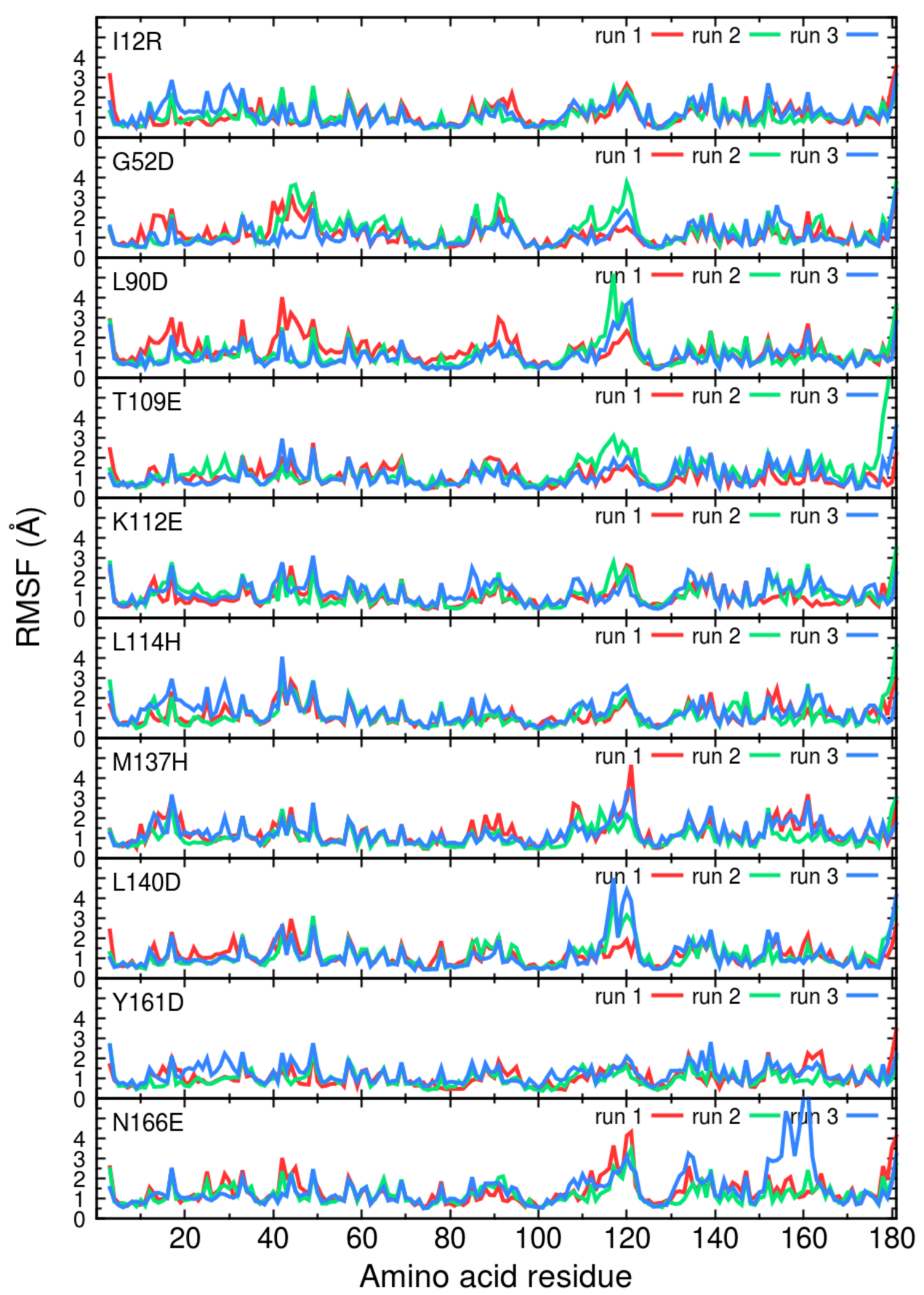

Figure S10. RMSF of each residue of BSLA beneficial substitutions determined from the last 40 ns of MD simulations in $12 \%(\mathrm{v} / \mathrm{v})$ TFE. Three independent MD runs are shown. 


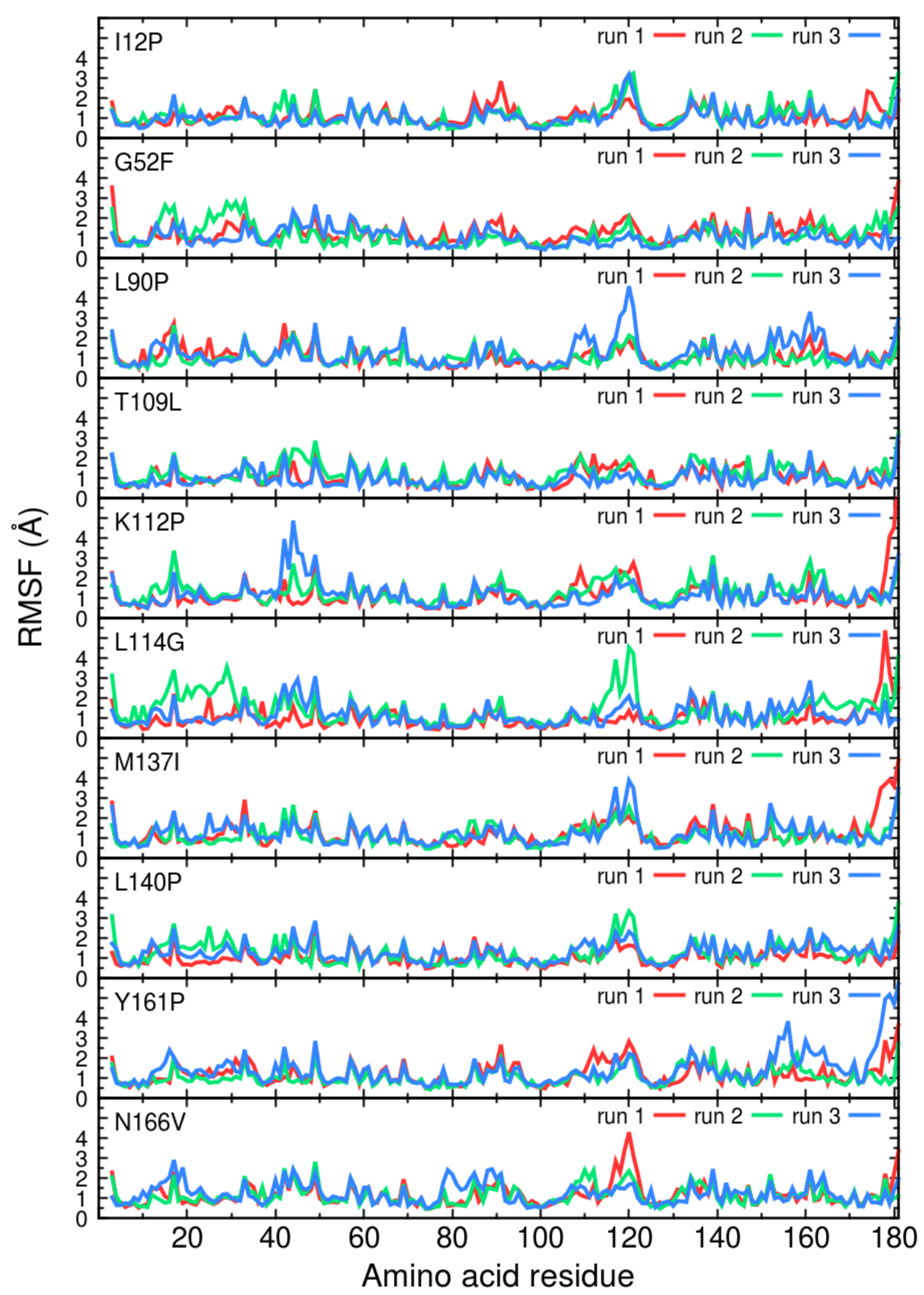

Figure S11. RMSF of each residue of BSLA non-beneficial substitutions determined from the last $40 \mathrm{~ns}$ of MD simulations in $12 \%(\mathrm{v} / \mathrm{v})$ TFE. Three independent MD runs are shown. 


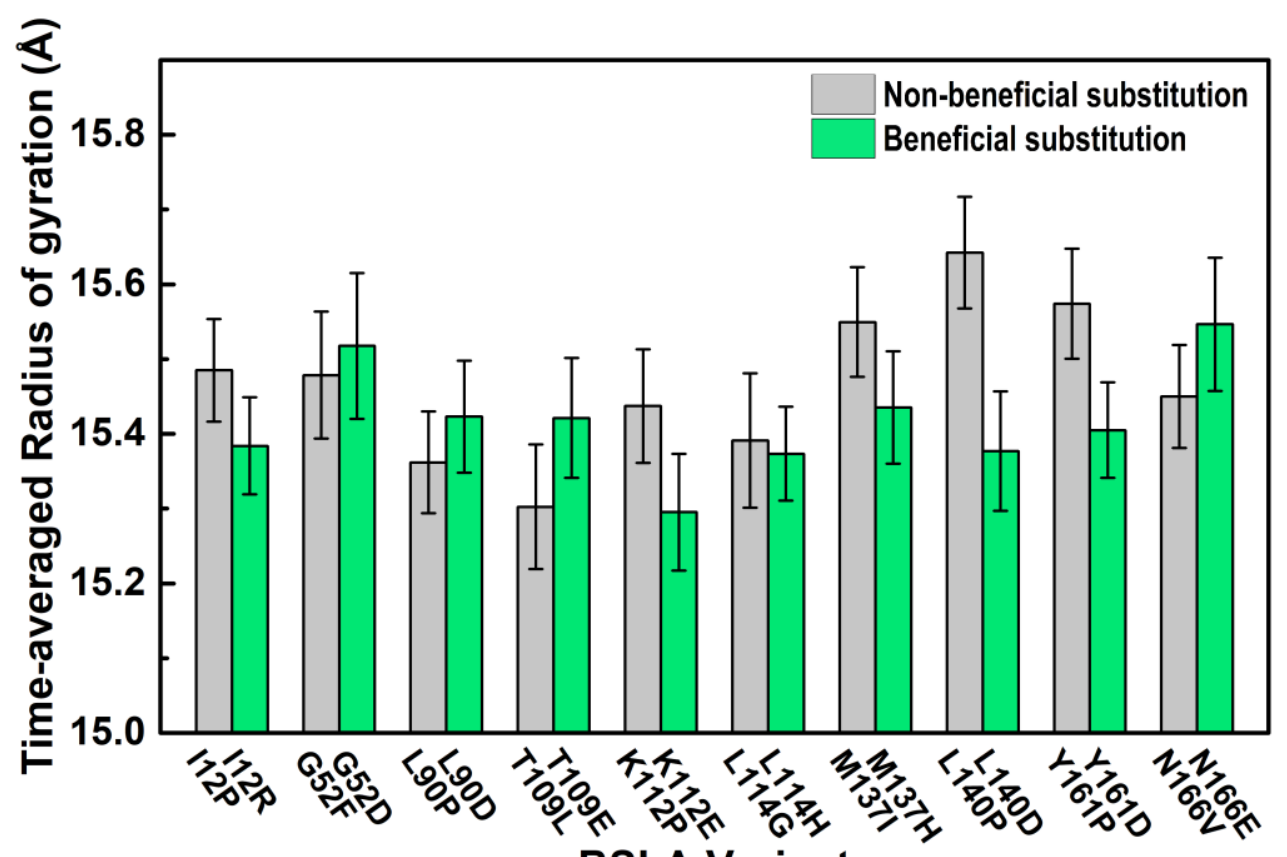

BSLA Variant

Figure S12. The time-averaged radius of gyration $\left(\mathrm{R}_{\mathrm{g}}\right)$ of BSLA beneficial and non-beneficial substitutions in $12 \%(\mathrm{v} / \mathrm{v})$ TFE, the time-averaged $\mathrm{R}_{\mathrm{g}}$ was calculated from the last $40 \mathrm{~ns}$ of the MD simulations. Error bars show the standard deviation from three independent MD runs. 


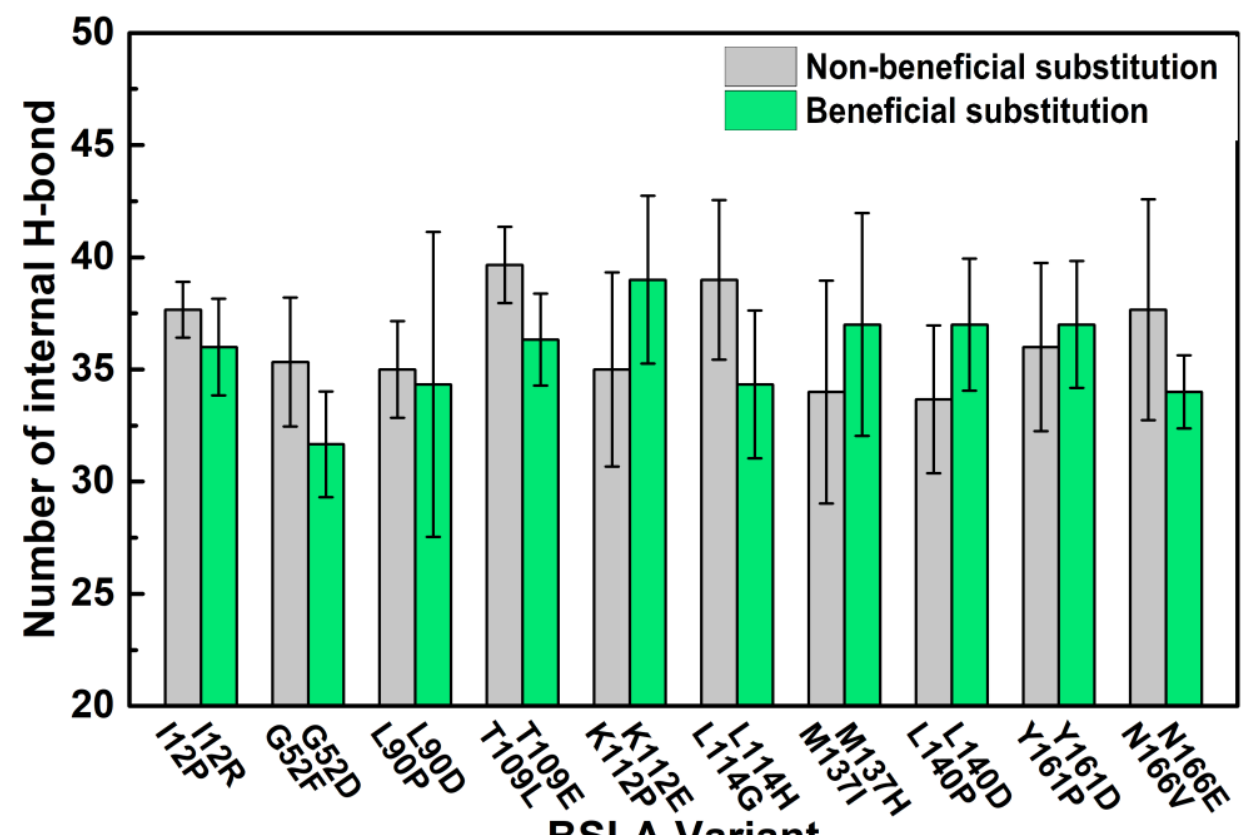

BSLA Variant

Figure S13. The number of internal hydrogen bonds with > 95\% occupancy in BSLA beneficial and non-beneficial substitutions. All the data were calculated from the last $40 \mathrm{~ns}$ of the simulation. Error bars show the standard deviation from three independent MD runs in $12 \%$ (v/v) TFE. Geometric cut off for evaluation of hydrogen bond distance $3.5 \AA$ and angle $30^{\circ}$ were used. 
(a)

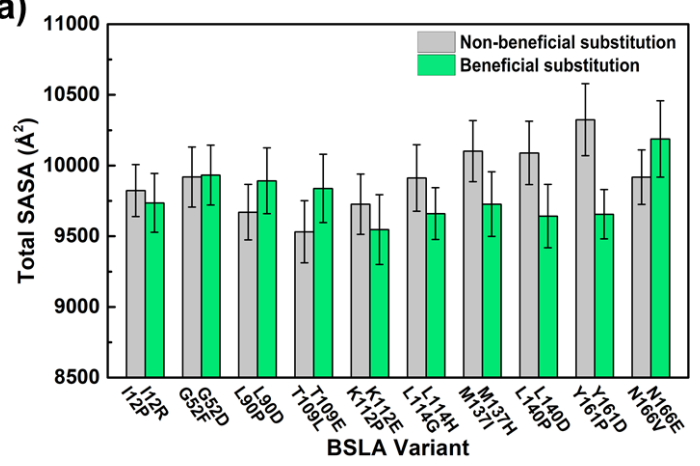

(c)

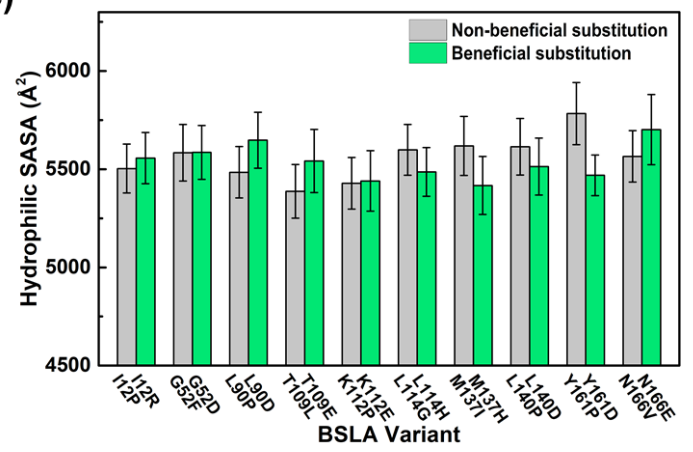

(b)

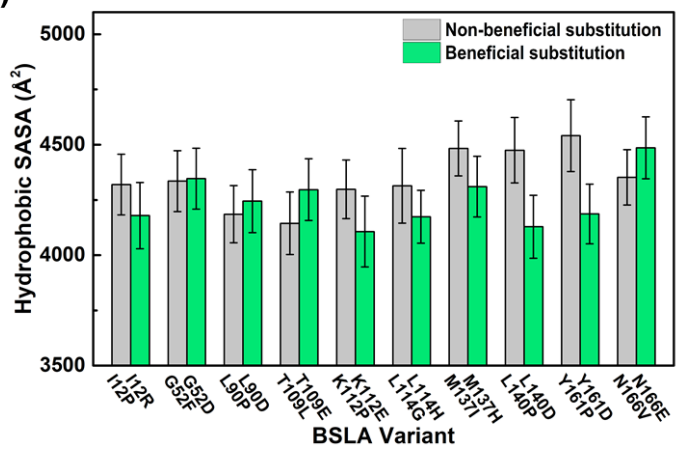

Figure S14. The time-averaged (a) total (b) hydrophobic (c) hydrophilic SASA of BSLA beneficial and non-beneficial substitutions. The average of SASA is computed based on the last $40 \mathrm{~ns}$ of each simulation. Error bars show the standard deviation from three independent MD runs for each variant. Here, SASA refers to the surface area of BSLA, which is accessible to water molecules and organic solvents molecules calculated using a probe of radius $1.4 \AA$. The cut off 0.2 to 0.2 was used for hydrophobic and hydrophilic SASA calculations ${ }^{9}$. 


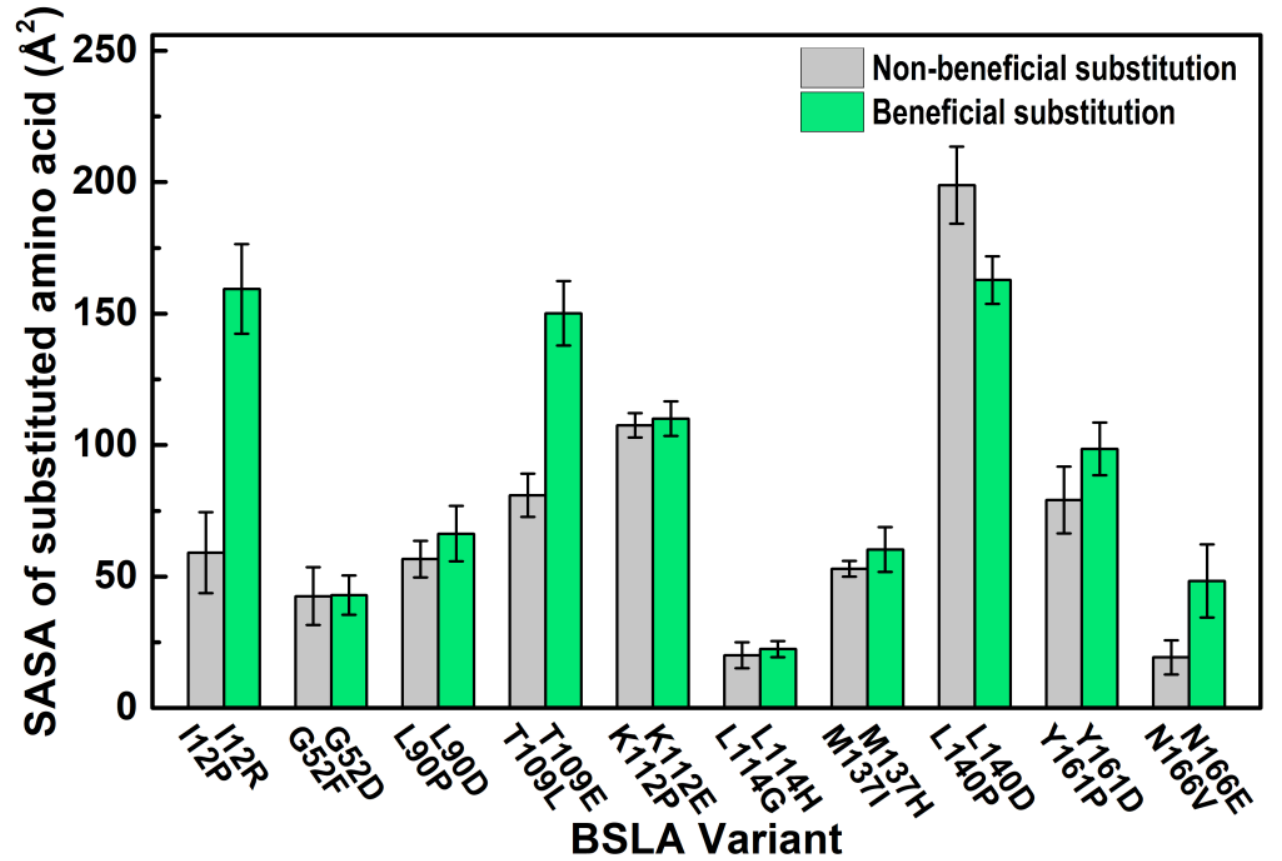

Figure S15. The SASA of the substituted residue of BSLA beneficial and non-beneficial substitutions in $12 \%(\mathrm{v} / \mathrm{v})$ TFE during MD simulations. The SASA of substituted residue was averaged over the last $40 \mathrm{~ns}$ of three independent simulations. 


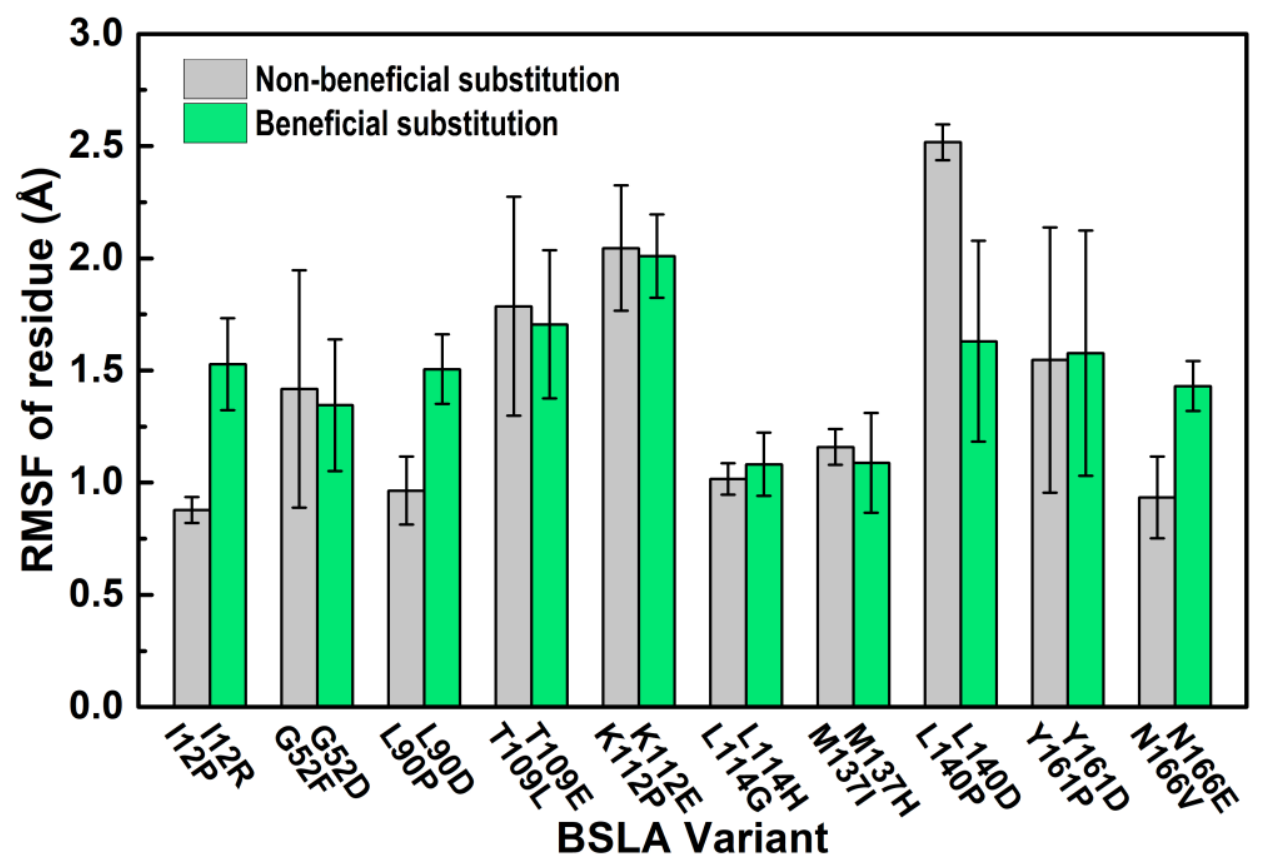

Figure S16. The RMSF of the substituted residue of BSLA beneficial and non-beneficial substitutions in $12 \%(\mathrm{v} / \mathrm{v})$ TFE during MD simulations. The RMSF of substituted residue was averaged over the last $40 \mathrm{~ns}$ of three independent simulations. 
(a)

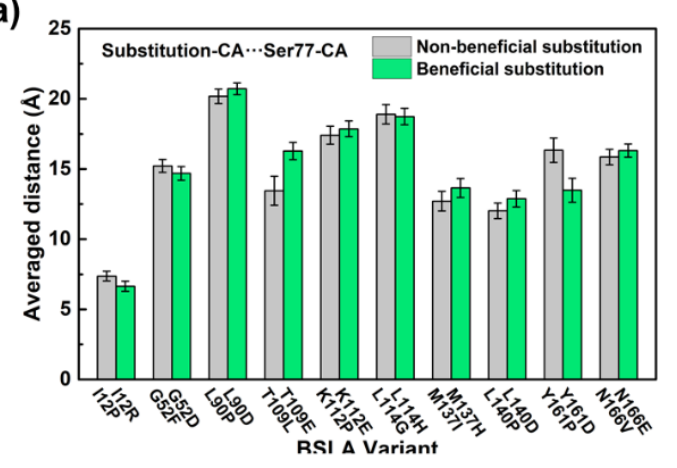

(c)

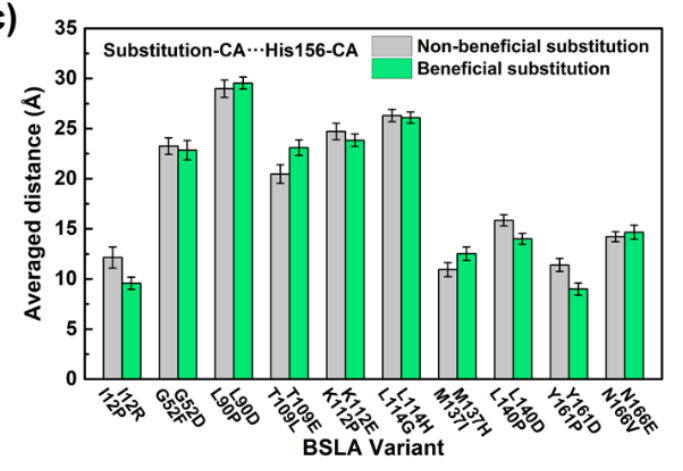

(b)

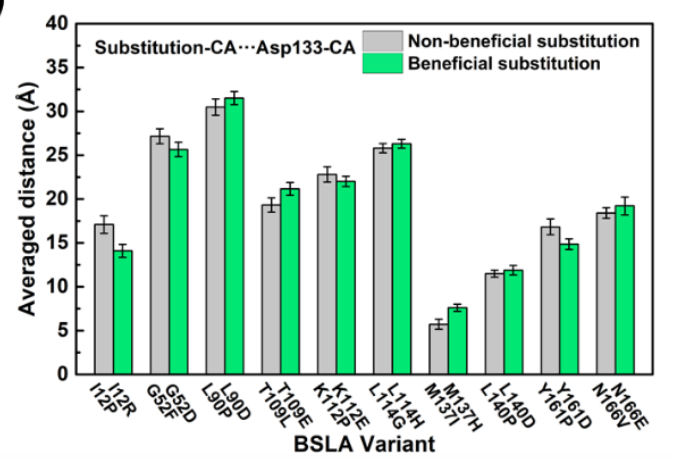

Figure S17. Averaged distance between substituted residue and the catalytic triad (a) Ser77, (b) Asp133, (c) His156 of BSLA beneficial and non-beneficial substitutions. The internal-atomic $\left(\mathrm{C}_{\alpha}\right.$ atom) distance $(\AA)$ is defined as the distance between substituted residue and the catalytic triad and averaged over the last $40 \mathrm{~ns}$ of three independent simulations. 
(a)

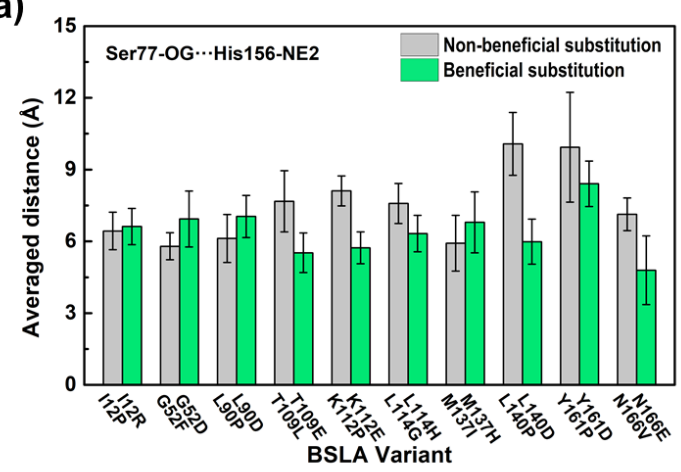

(c)

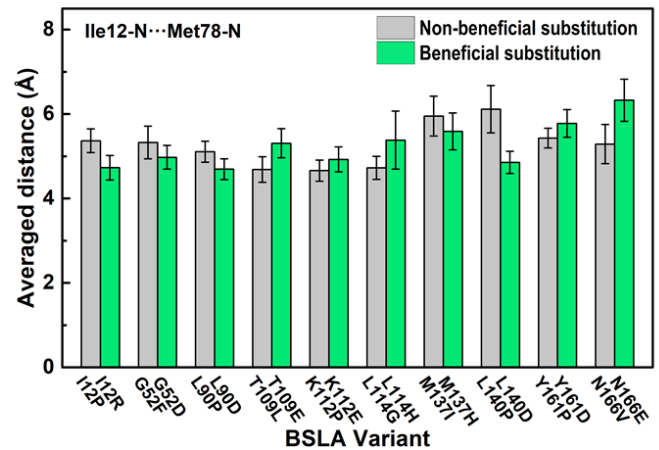

(b)

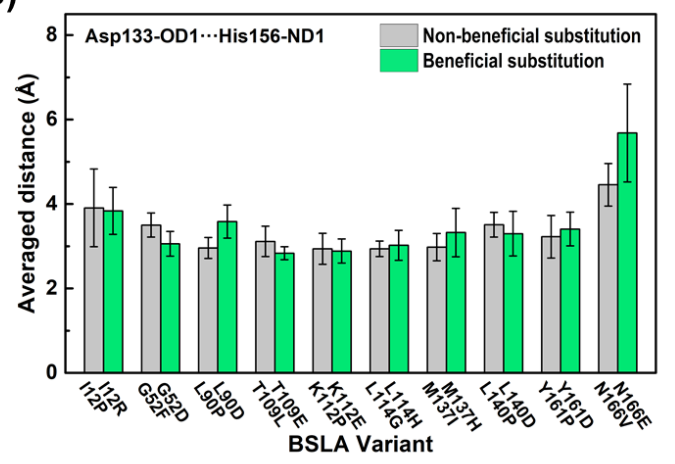

(d)

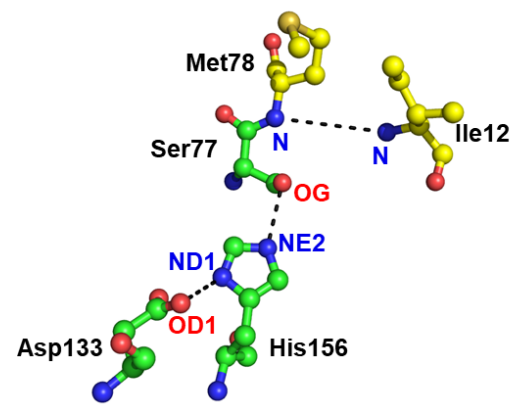

Figure S18. The averaged internal-atomic distance between the catalytic triad (Ser77, His156, Asp133) and oxyanion hole (Ile12, Met78) of BSLA beneficial and non-beneficial substitutions:

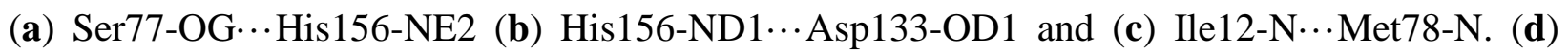
Representative structures of the catalytic triad and oxyanion hole were taken from BSLA WT (PDB ID: $116 w^{10}$, Chain A). The catalytic triad and oxyanion hole are shown as ball-and-stick with carbon (green/yellow), oxygen (red), and nitrogen (blue). The dashed line indicates the distance between two atoms. 
(a)

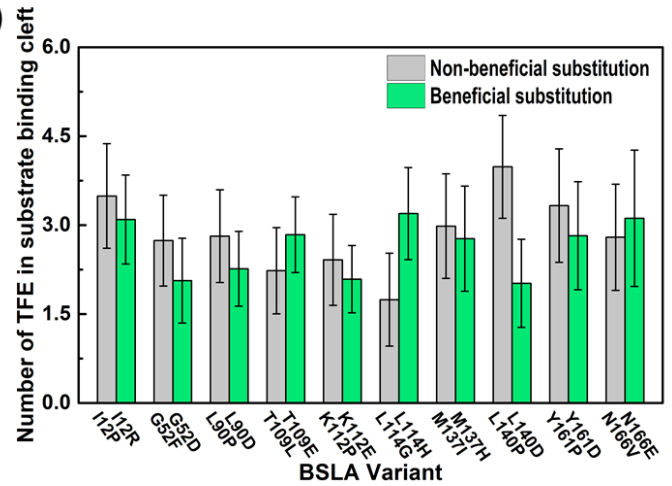

(b) $\frac{\mathrm{c}}{\mathrm{c}}$

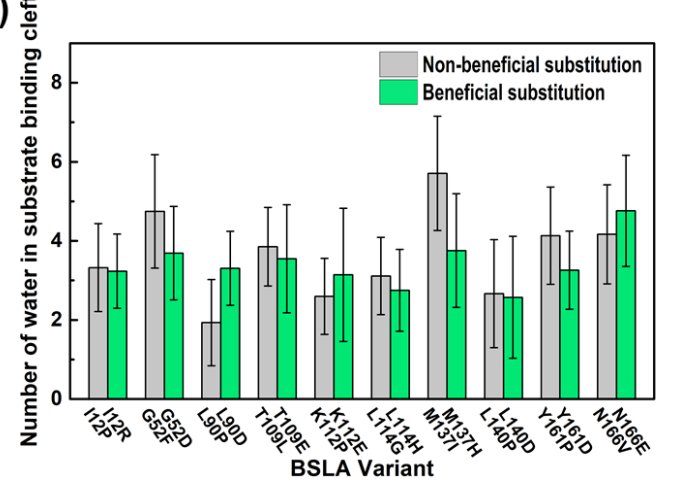

Figure S19. The average number of solvent molecules in substrate binding cleft of BSLA beneficial and non-beneficial substitutions during the MD simulations: (a) TFE, and (b) water. The number of water/TFE molecules was averaged over the last $40 \mathrm{~ns}$ from three independent MD runs. 


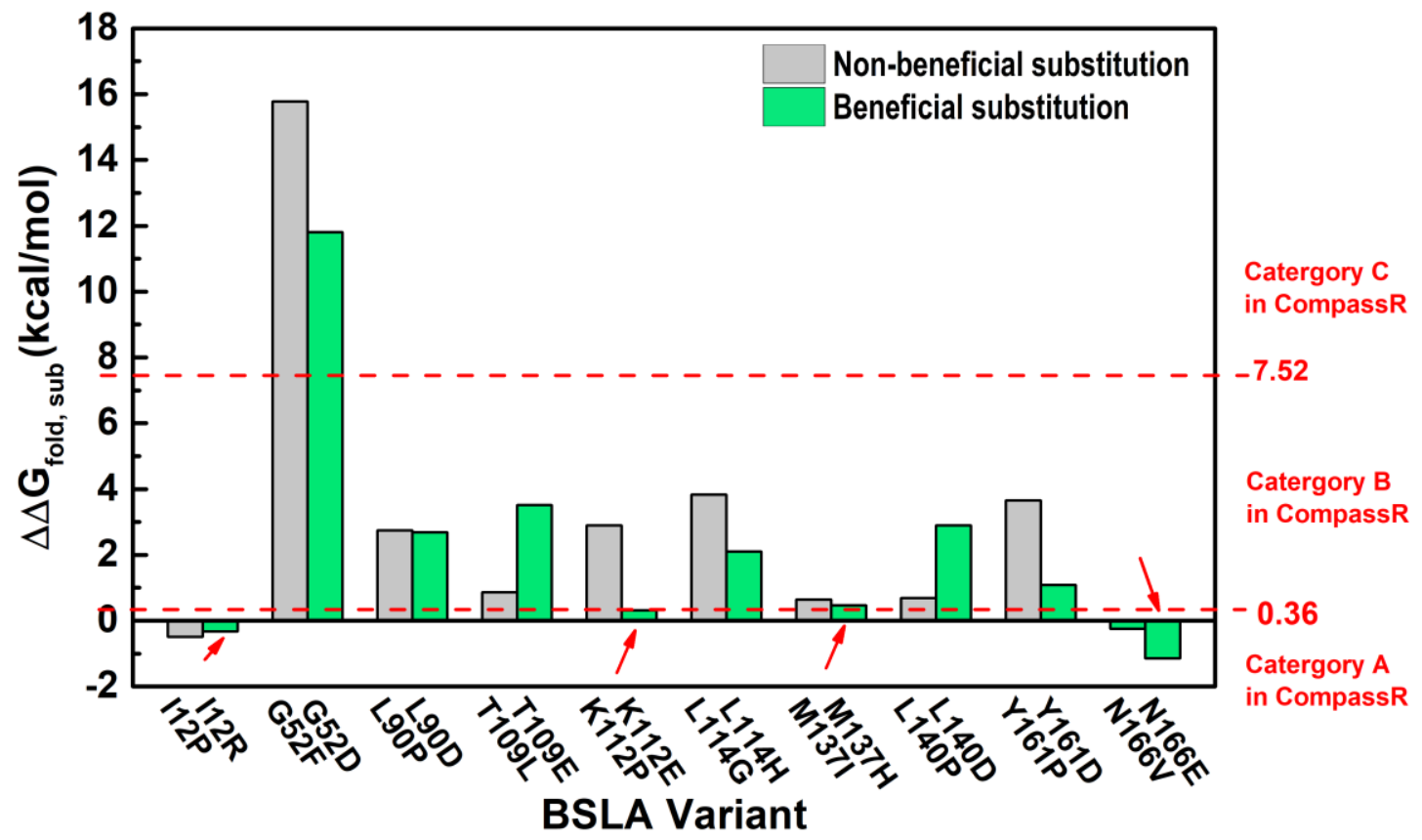

Figure S20. $\Delta \Delta G_{\text {fold }}$ values of 20 BSLA substitutions at ten positions and grouped into three categories according to CompassR rule. CompassR rule: when substitutions with $\Delta \Delta G_{\text {fold }}$ values $\leq$ $+0.36 \mathrm{kcal} / \mathrm{mol}$ are recombined one can expect active and property improved recombinants; when beneficial substitutions are recombined with $\Delta \Delta G_{\text {fold }}$ values ranging from +0.36 to $+7.52 \mathrm{kcal} / \mathrm{mol}$ one cannot predict whether the recombinants will be inactive or active (unpredictable behavior); recombination of beneficial substitutions with $\Delta \Delta G_{\text {fold }} \geq+7.52 \mathrm{kcal} / \mathrm{mol}$ results in deactivated and in activity-reduced recombinants. The relative folding free energies $\left(\Delta \Delta G_{\text {fold }}=\Delta G_{\text {fold,sub }}-\Delta G_{\text {fold,wt }}\right)$ were computed using FoldX $4{ }^{11}$ employing YASARA Plugin ${ }^{12}$ in YASARA Structure version 17.4.17 ${ }^{13}$ as our previous reported ${ }^{14}$. The larger the $\Delta \Delta G_{\text {fold }}$ negative values, the higher the stability. 


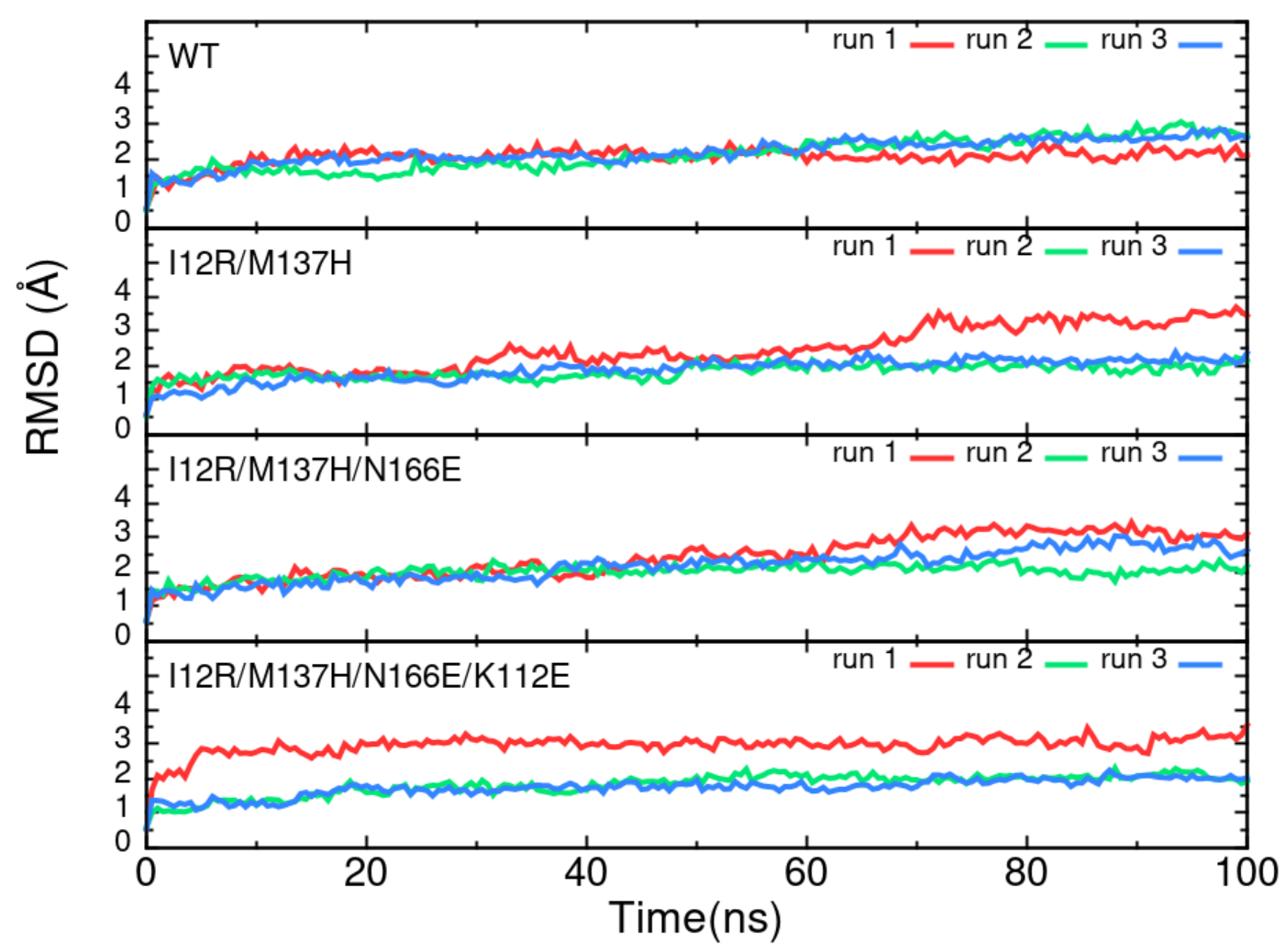

Figure S21. Backbone root-mean-square deviation (RMSD) of the BSLA WT and recombinants with respect to the initial structure as a function of time in $12 \%(\mathrm{v} / \mathrm{v})$ TFE. Three independent MD runs for each solvent are shown. 
(a) I12P

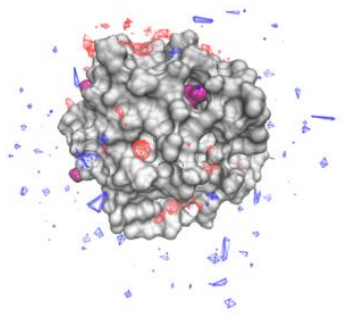

(e) I12R/M137H/N166E

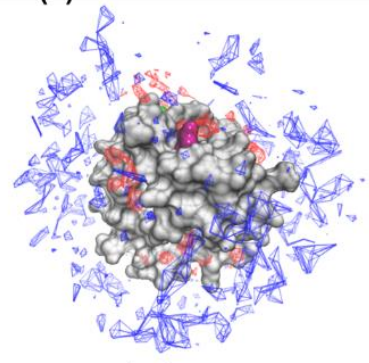

(b) WT

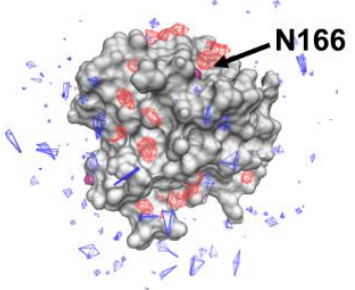

(f) I12R/M137H/N166E/K112E

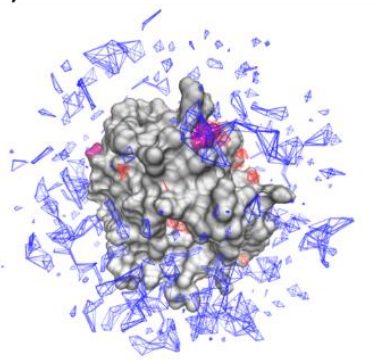

(c) I12R

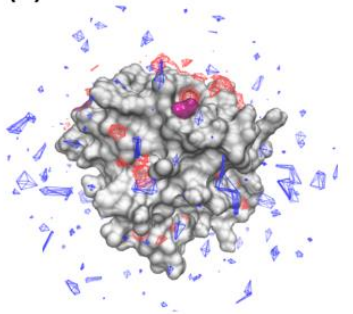

(d) I12R/M137H

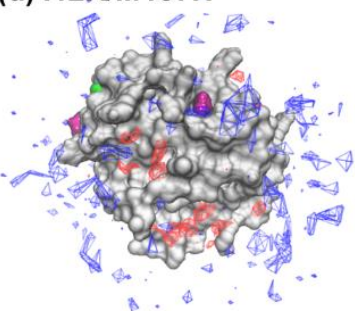

(g)

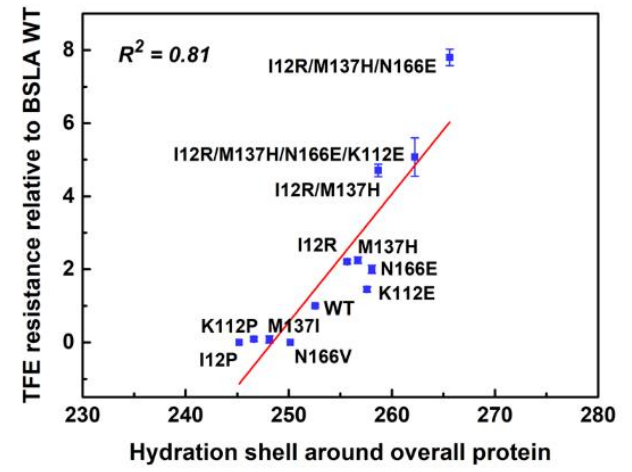

Figure S22. Spatial distribution of water and TFE occupancy at the surface of the BSLA variant (a) $\mathrm{I} 12 \mathrm{P}, \quad$ (b) $\mathrm{WT}$ I12R/M137H/N166E/K112E in $12 \%$ (v/v) TFE. The BSLA surface is shown in grey, S77, D133, H156 (the catalytic triad) in green, the OS molecules in red with wireframe, the water molecules in blue with the wireframe. The four substituted sites I12, K112, M137, N166, are labeled and shown in purple. A $180^{\circ}$ rotation view around the vertical axis is shown in Figure 4 to give a complete view of the surface. The three-dimensional arrangement (denoted as solvent densities) of water and TFE molecules around BSLA were calculated in terms of the spatial distribution function (SDF). SDF of water and TFE molecules were calculated using the GROMACS analysis tools (gmx spatial command) in all the systems of BSLA variants. Each view of the BSLA variant has the same orientation. The contours are shown with the isovalue 60, 75 for water, TFE molecules, respectively. (g) Variation of TFE resistance relative to BSLA WT as a function of hydration level around overall protein; $R^{2}=0.81$. The total number of water molecules whose $\mathrm{O}$ atom is within $3.5 \AA$ distance cut-off of residue 12, 137, 166, and 112 were described as hydration level around substituted residues ${ }^{4,15}$. Hydration level averaged over the last $40 \mathrm{~ns}$ from three independent MD runs. 
(a)

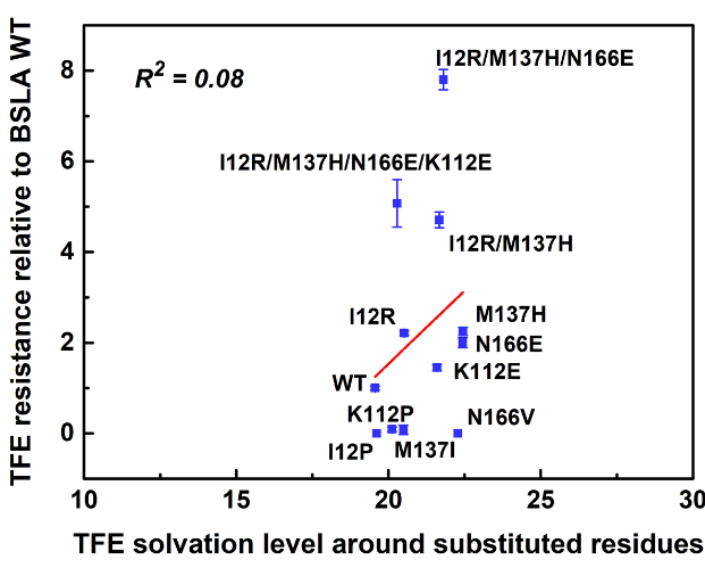

(b)

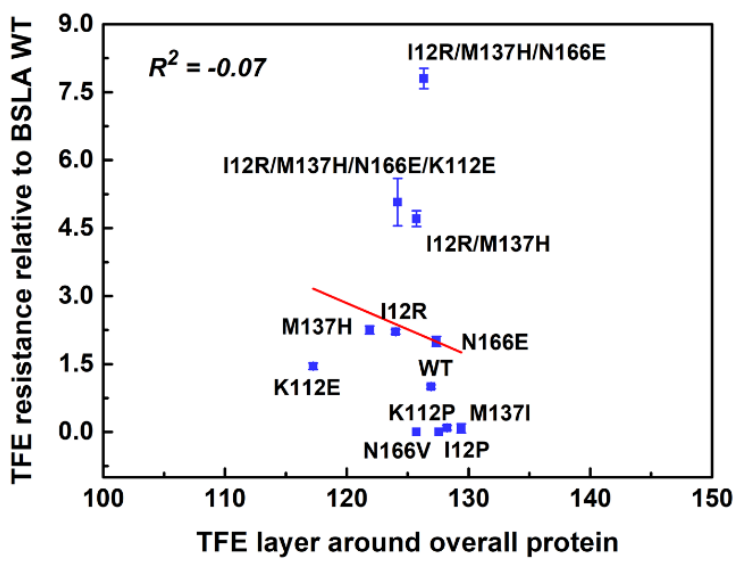

Figure S23. Variation of TFE resistance relative to BSLA WT as a function of (a) TFE layer around overall protein; $R^{2}=-0.07$, (b) TFE solvation level around substituted residues; $R^{2}=0.08$. The $6.8 \AA$ was selected as a cut-off to define the TFE layer, which was determined from the radial distribution function (RDF) of TFE around BSLA residues when the "central" atom C2 of TFE molecule showed first minima approximately at this distance ${ }^{4}$. The number of solvent molecules was averaged over the last $40 \mathrm{~ns}$ from three independent MD runs. 


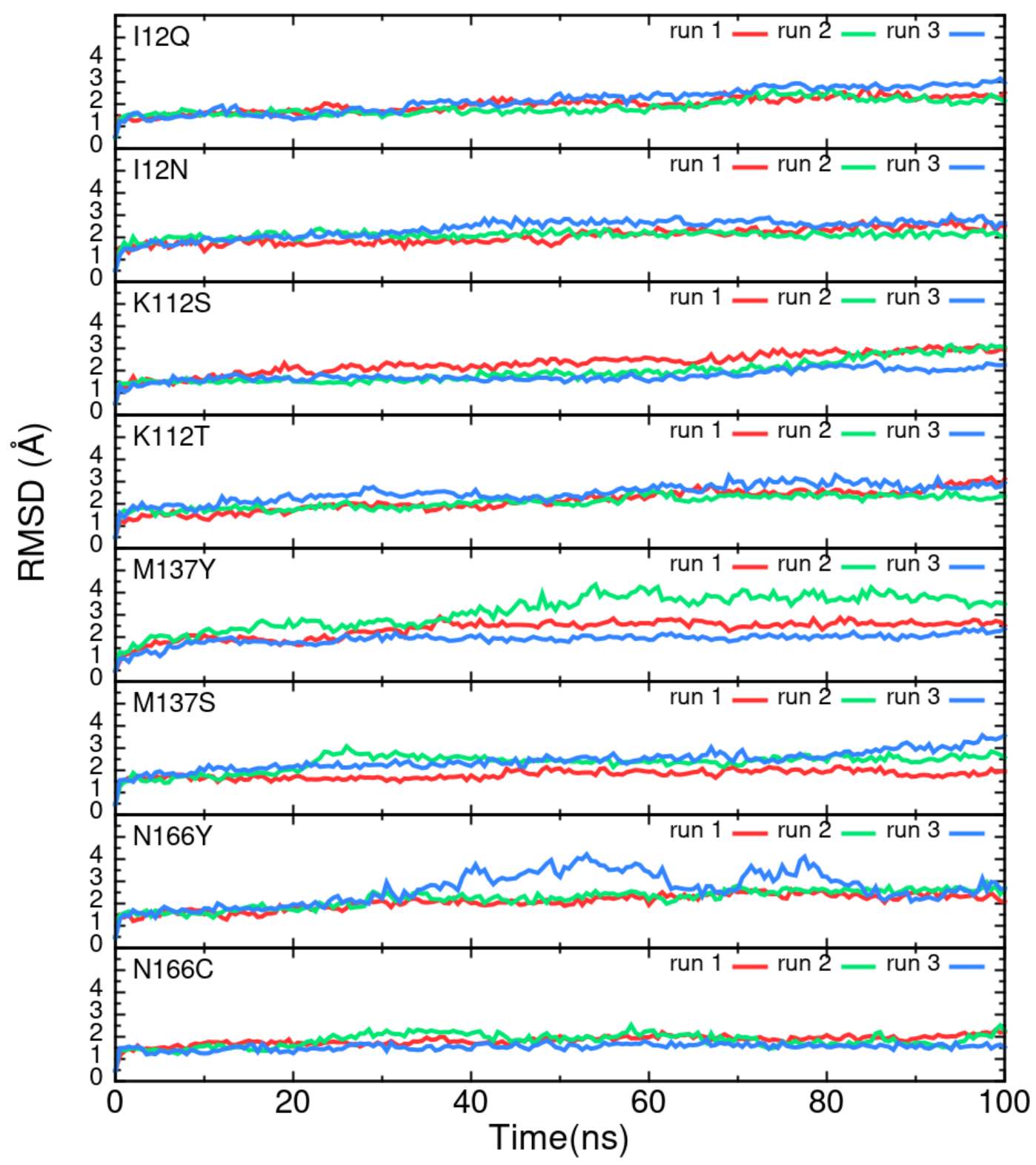

Figure S24. Backbone root-mean-square deviation (RMSD) of the BSLA polar substitutions with respect to the initial structure as a function of time in $12 \%(\mathrm{v} / \mathrm{v})$ TFE. Three independent MD runs for each solvent are shown. 
(a)

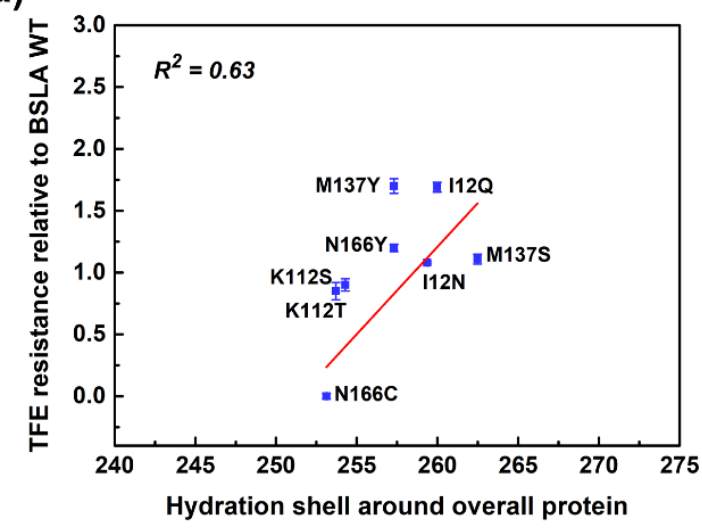

(c)

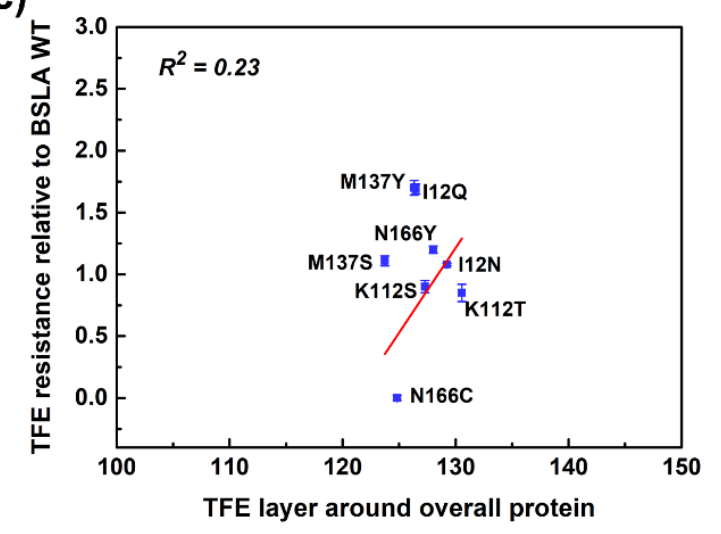

(b)

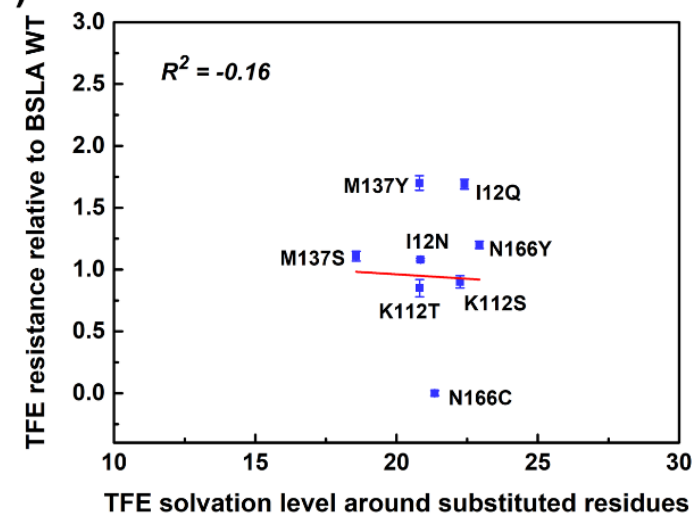

Figure S25. Solvation phenomenon of BSLA polar substitutions. Variation of TFE resistance relative to BSLA WT as a function of (a) hydration shell around overall protein, $R^{2}=0.63$. Variation of TFE resistance relative to BSLA WT as a function of (b) TFE solvation level around substituted residues, $R^{2}=-0.16$, and (c) TFE layer around overall protein, $R^{2}=0.23$. The $3.5 \AA$ was selected as a cut-off to define the hydration shell around overall protein ${ }^{4,15}$. The $6.8 \AA$ was selected as a cut-off to define the TFE layer, which was determined from the radial distribution function (RDF) of TFE around BSLA residues when the "central" atom C2 of TFE molecule showed first minima approximately at this distance ${ }^{4}$. The number of solvent molecules was averaged over the last $40 \mathrm{~ns}$ from three independent MD runs. 


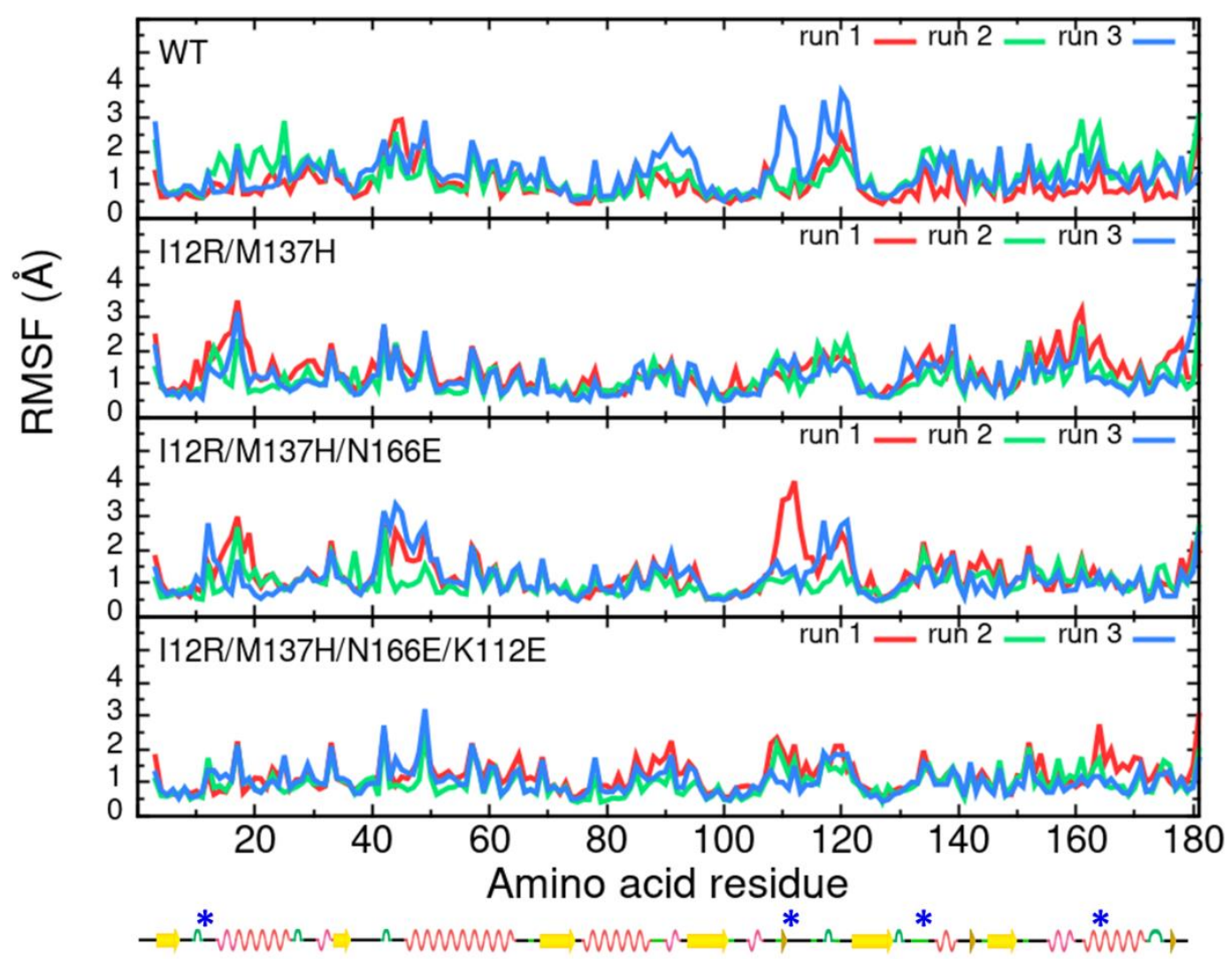

Figure S26. RMSF of each residue of BSLA WT and recombinants determined from the last 40 ns of MD simulations in $12 \%(\mathrm{v} / \mathrm{v})$ TFE. The secondary structure elements were calculated using the DSSP program ${ }^{16}$. Secondary structure elements are shown by the following color scheme: $\beta$ sheet (yellow), $\alpha$-helix (pink), 3/10-helix (red), and turn, $\beta$-bridge, and bend (green). Substituted amino acid positions $(12,112,137,166)$ are labeled with asterisks. Three independent MD runs are shown. 


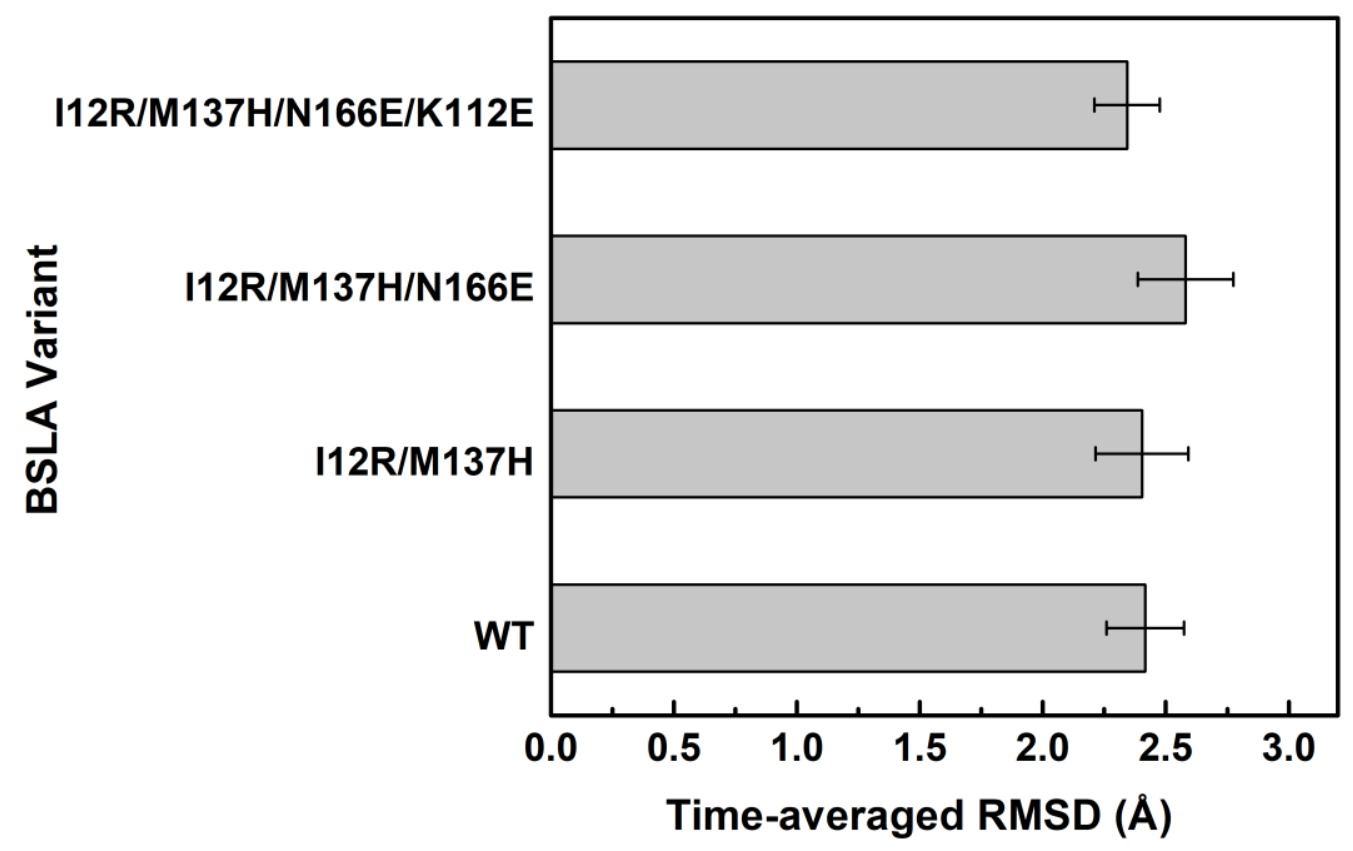

Figure S27. Time-average RMSD of BSLA WT and recombinants determined from the last $40 \mathrm{~ns}$ of simulations in $12 \%(\mathrm{v} / \mathrm{v})$ TFE. Error bars describe the standard deviation from three independent MD runs. 


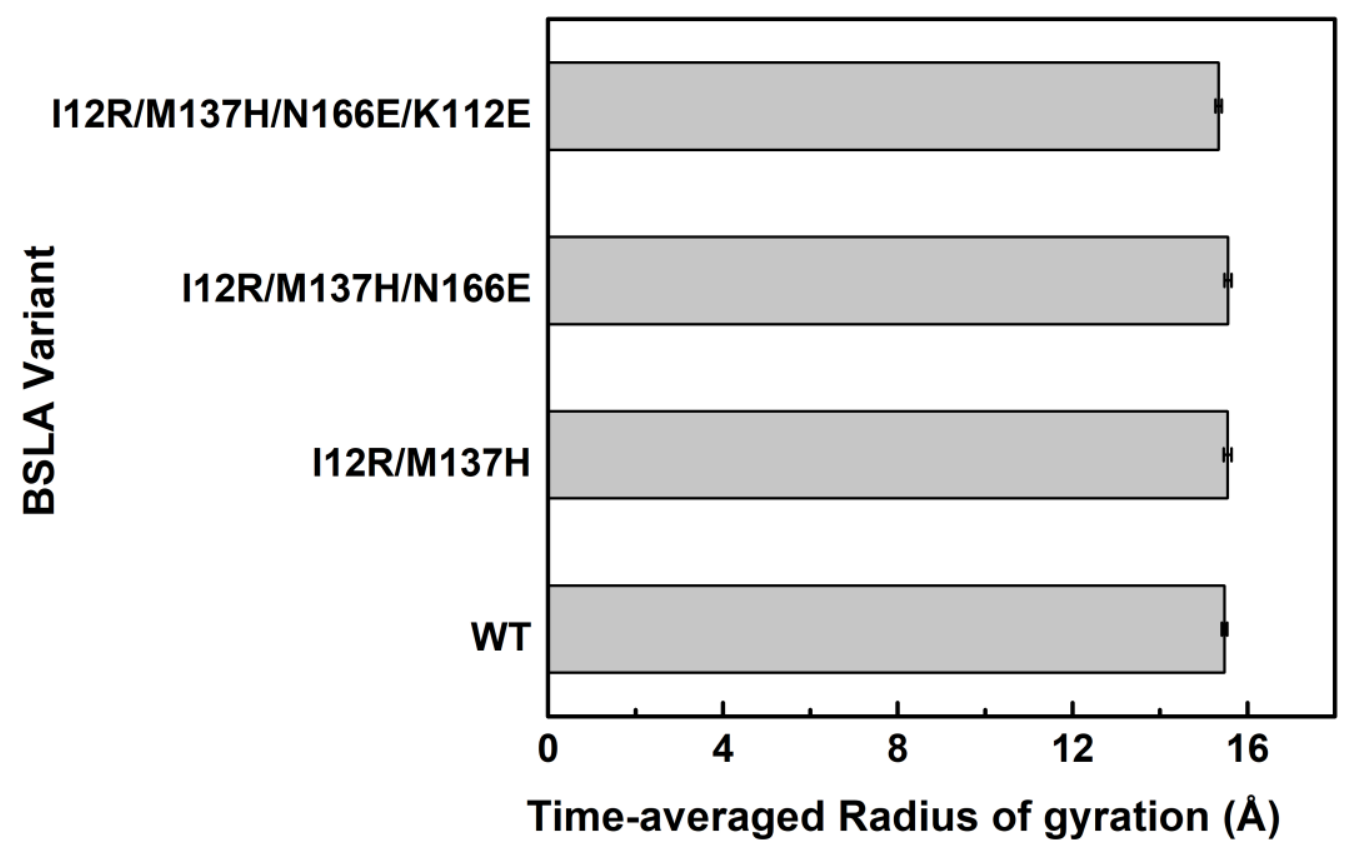

Figure S28. The time-averaged radius of gyration $\left(\mathrm{R}_{\mathrm{g}}\right)$ of BSLA WT and recombinants in $12 \%$ (v/v) TFE, the time-averaged $\mathrm{R}_{\mathrm{g}}$ was calculated from the last $40 \mathrm{~ns}$ of the MD simulations. Error bars show the standard deviation from three independent MD runs. 


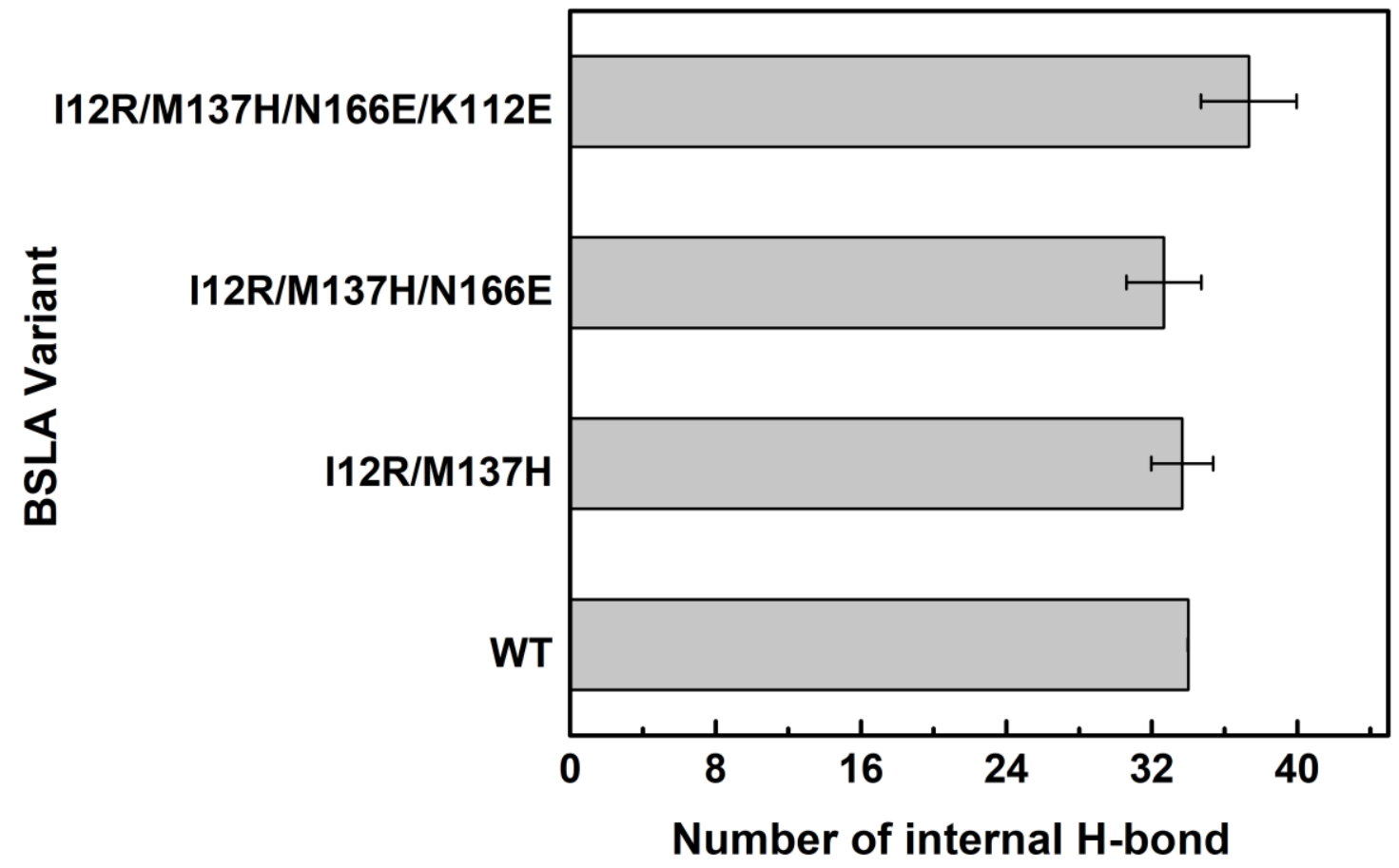

Figure S29. The number of internal hydrogen bonds with $>95 \%$ occupancy of BSLA WT and recombinants. All the data were calculated from the last $40 \mathrm{~ns}$ of the simulation. Error bars show the standard deviation from three independent MD runs in $12 \%(\mathrm{v} / \mathrm{v}) \mathrm{TFE}$. Geometric cut off for evaluation of hydrogen bond distance $3.5 \AA$ and angle $30^{\circ}$ were used. 


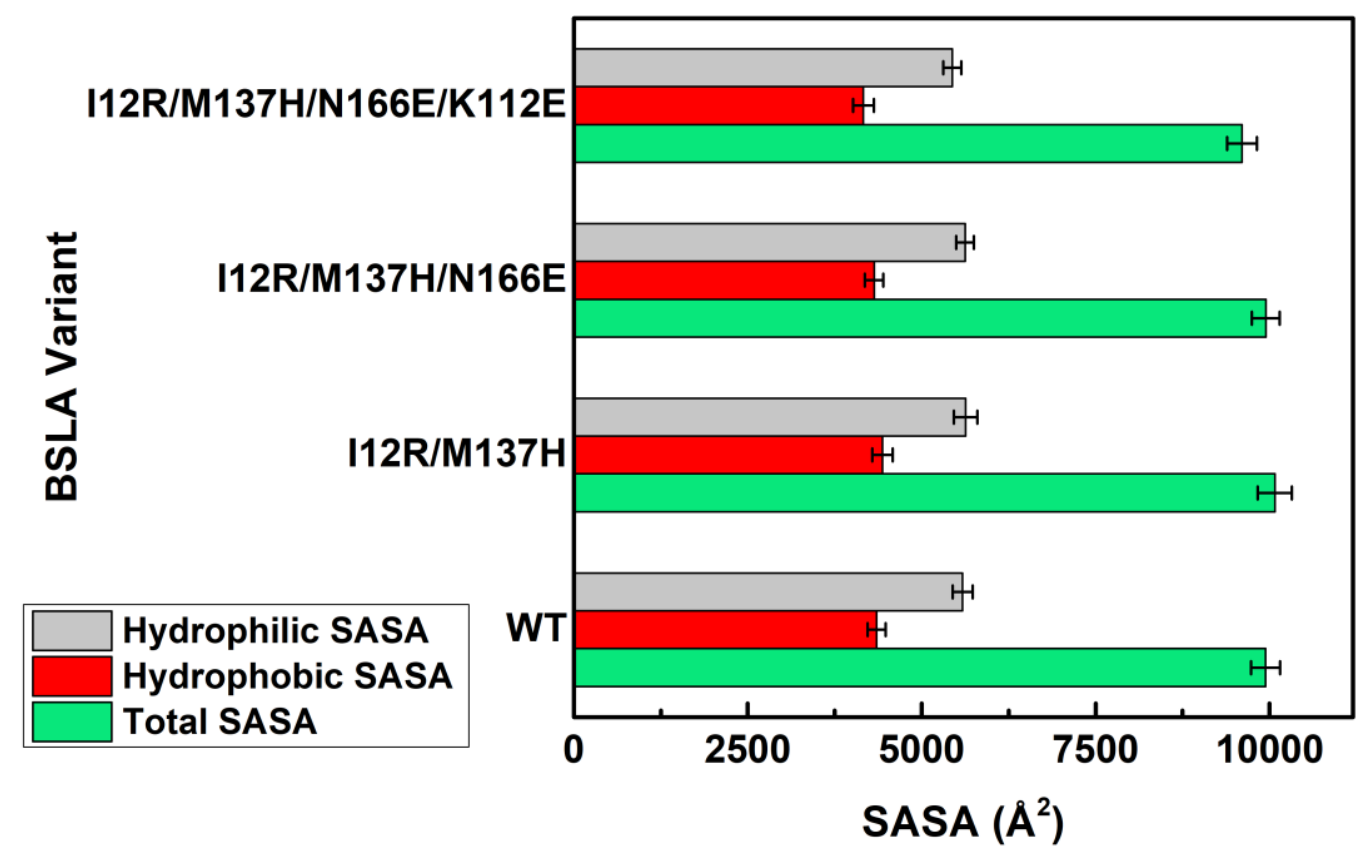

Figure S30. The time-averaged total, hydrophobic, hydrophilic SASA of BSLA WT and recombinants. The average of SASA is computed based on the last $40 \mathrm{~ns}$ of each simulation. Error bars show the standard deviation from three independent MD runs for each variant. Here, SASA refers to the surface area of BSLA, which is accessible to water molecules and organic solvents molecules calculated using a probe of radius $1.4 \AA$. The cut off -0.2 to 0.2 was used for hydrophobic and hydrophilic SASA calculations ${ }^{9}$. 


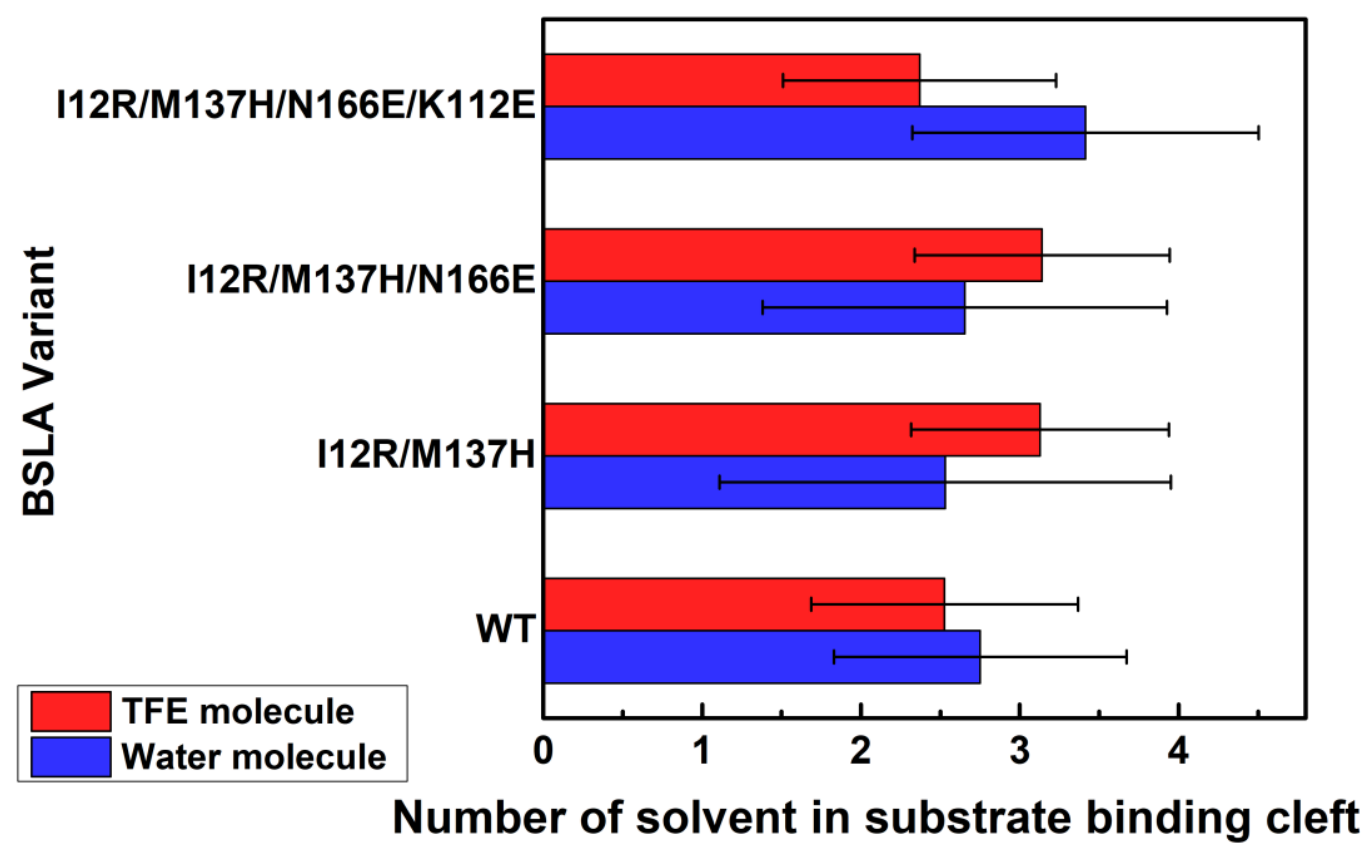

Figure S31. The average number of TFE and water molecule in substrate binding cleft of BSLA WT and recombinants during the MD simulations. The number of water/TFE molecules was averaged over the last $40 \mathrm{~ns}$ from three independent MD runs. 


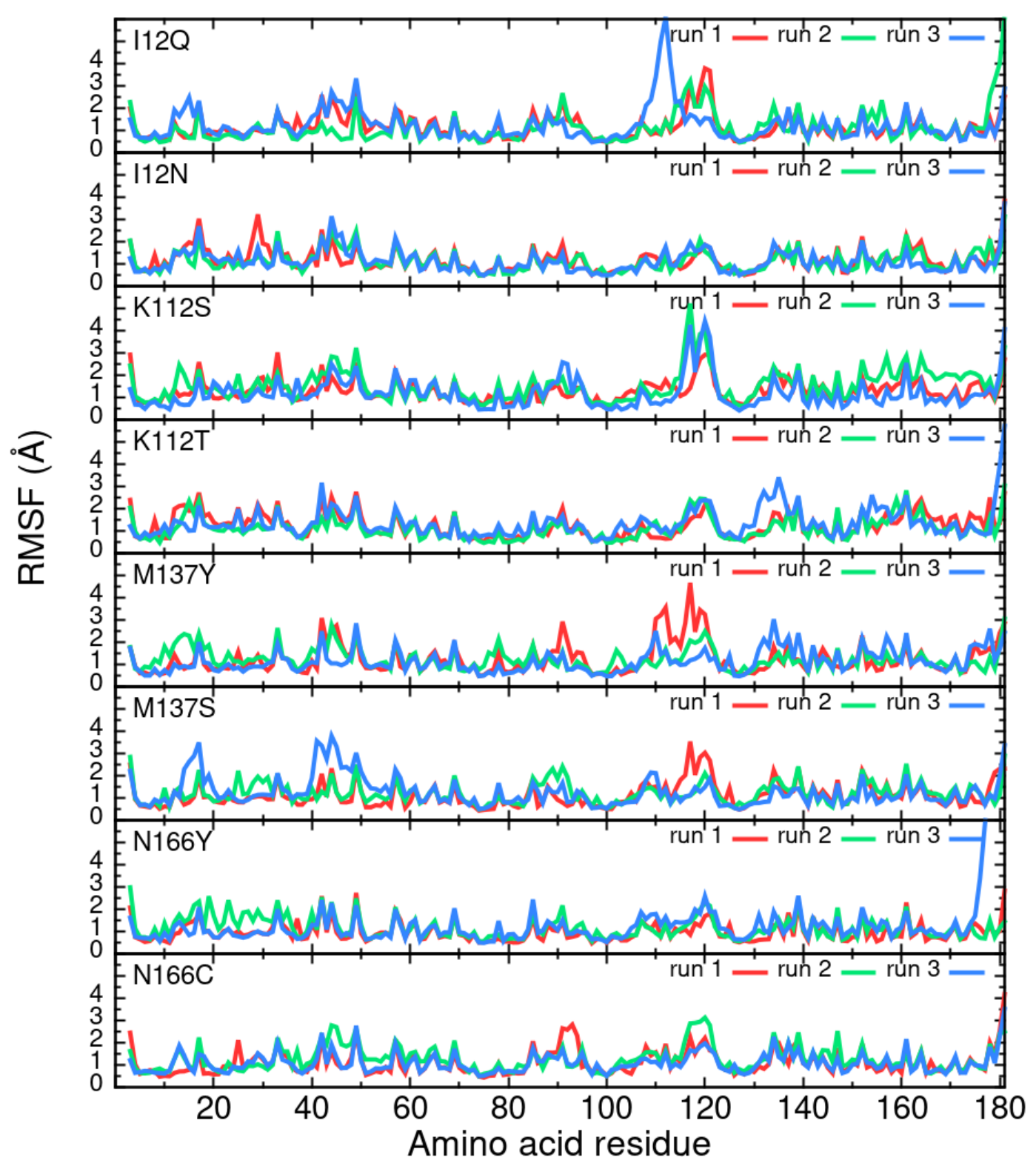

Figure S32. RMSF of each residue of BSLA WT and recombinants determined from the last 40 ns of MD simulations in $12 \%(\mathrm{v} / \mathrm{v})$ TFE. The secondary structure elements were calculated using the DSSP program ${ }^{16}$. Secondary structure elements are shown by the following color scheme: $\beta$ sheet (yellow), $\alpha$-helix (pink), 3/10-helix (red), and turn, $\beta$-bridge, and bend (green). Substituted amino acid positions $(12,112,137,166)$ are labeled with asterisks. Three independent MD runs are shown. 


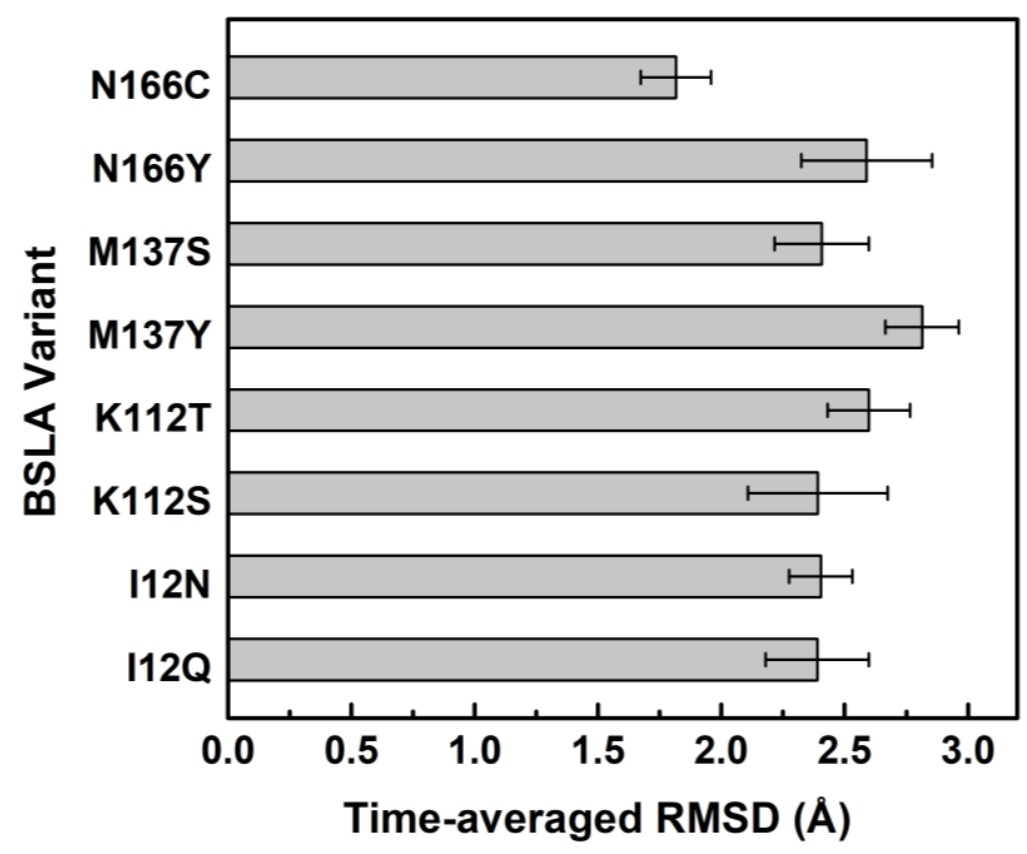

Figure S33. Time-average RMSD of BSLA polar substitutions determined from the last $40 \mathrm{~ns}$ of simulations in $12 \%$ (v/v) TFE. Error bars describe the standard deviation from three independent MD runs. 


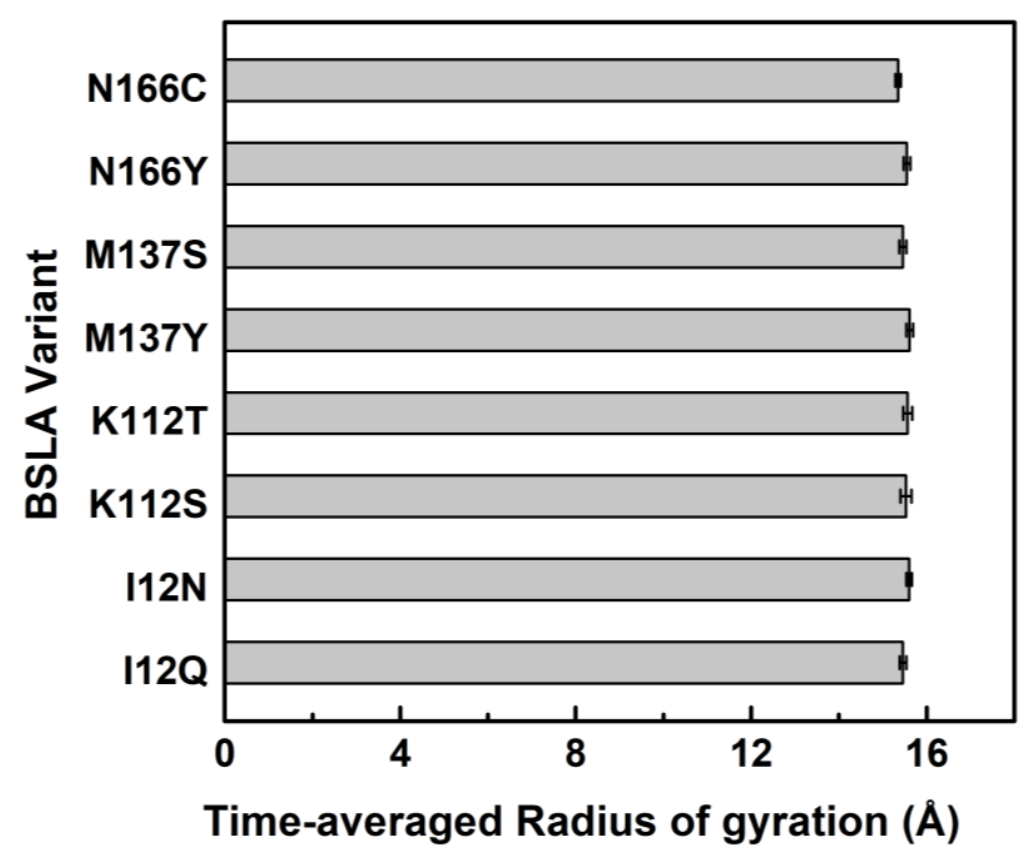

Figure S34. The time-averaged radius of gyration $\left(\mathrm{R}_{\mathrm{g}}\right)$ of BSLA polar substitutions in $12 \%(\mathrm{v} / \mathrm{v})$ TFE, the time-averaged $\mathrm{R}_{\mathrm{g}}$ was calculated from the last $40 \mathrm{~ns}$ of the MD simulations. Error bars show the standard deviation from three independent MD runs. 


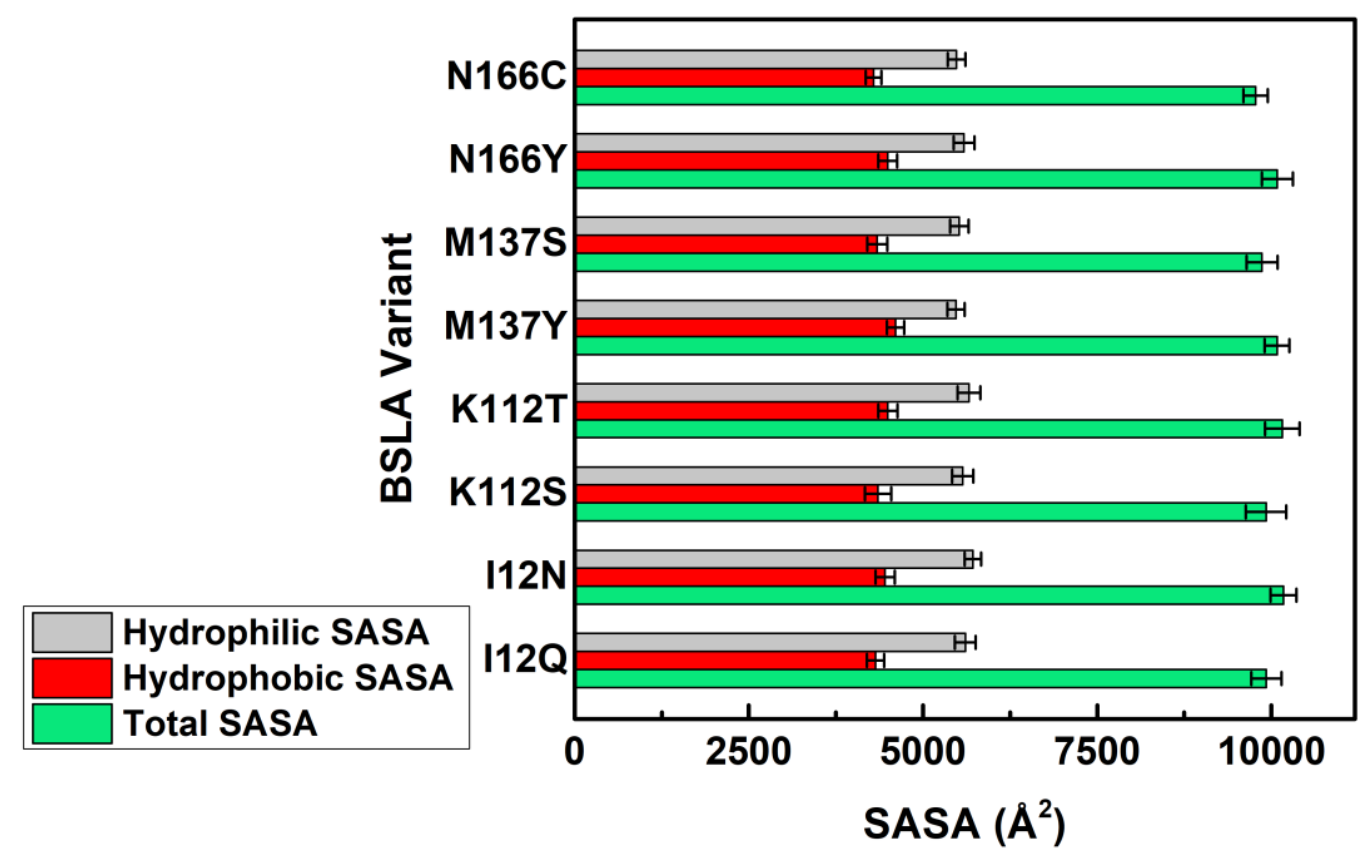

Figure S35. The time-averaged total, hydrophobic, hydrophilic SASA of BSLA polar substitutions. The average of SASA is computed based on the last $40 \mathrm{~ns}$ of each simulation. Error bars show the standard deviation from three independent MD runs for each variant. Here, SASA refers to the surface area of BSLA, which is accessible to water molecules and organic solvents molecules calculated using a probe of radius $1.4 \AA$. The cut off -0.2 to 0.2 was used for hydrophobic and hydrophilic SASA calculations ${ }^{9}$. 


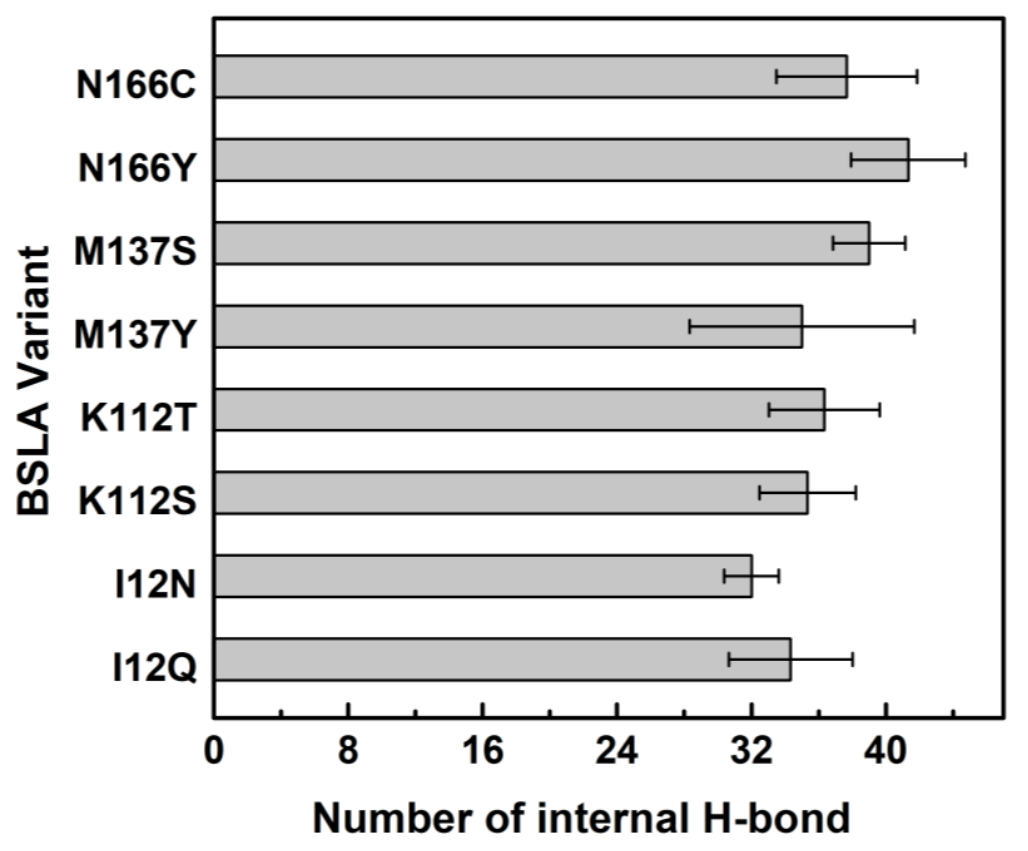

Figure S36. The number of internal hydrogen bonds with > 95\% occupancy of BSLA polar substitutions. All the data were calculated from the last $40 \mathrm{~ns}$ of the simulation. Error bars show the standard deviation from three independent MD runs in $12 \%(\mathrm{v} / \mathrm{v})$ TFE. Geometric cut off for evaluation of hydrogen bond distance $3.5 \AA$ and angle $30^{\circ}$ were used. 


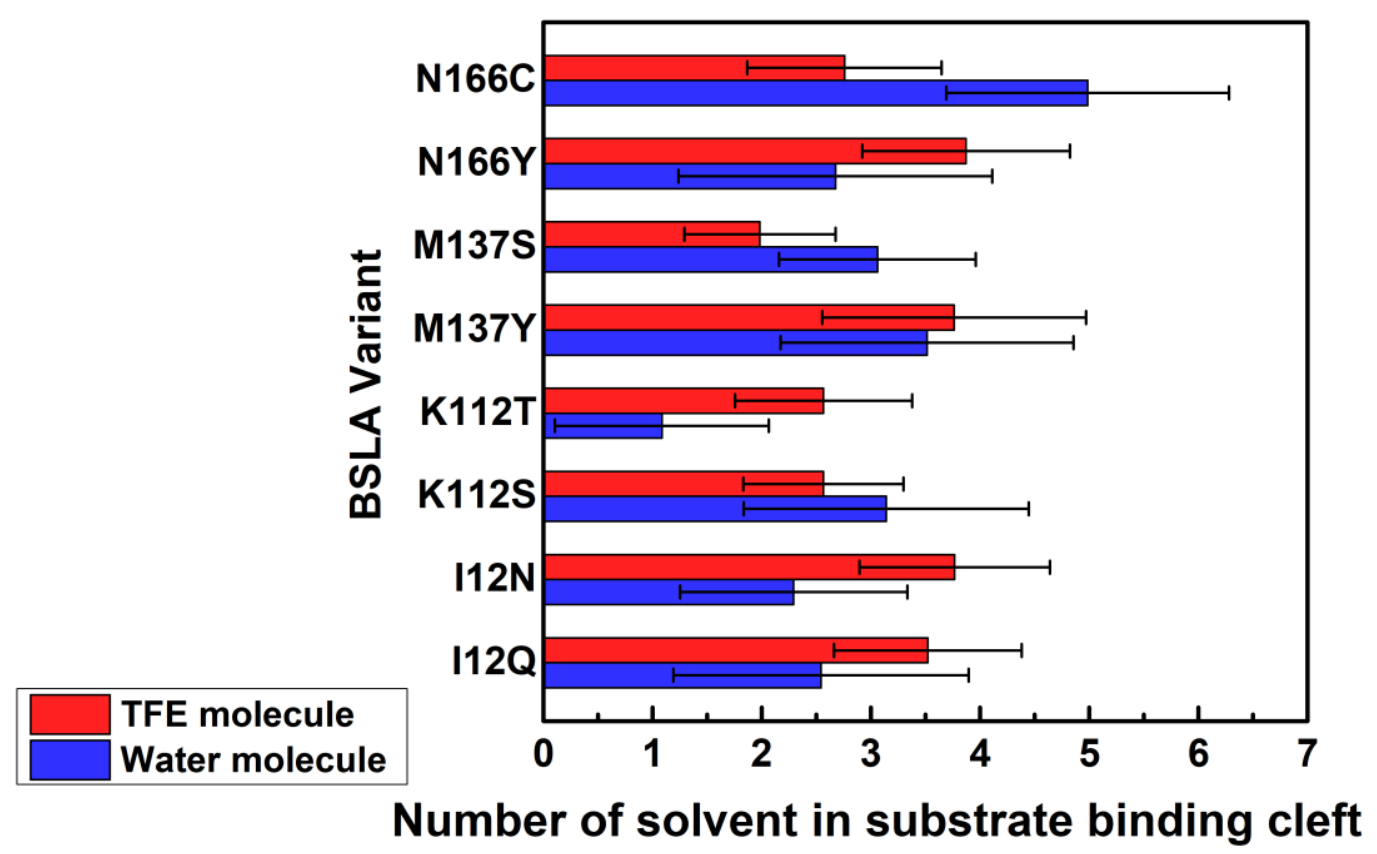

Figure S37. The average number of TFE and water molecule in substrate binding cleft of BSLA polar substitutions during the MD simulations. The number of water/TFE molecules was averaged over the last $40 \mathrm{~ns}$ from three independent MD runs. 
(a)

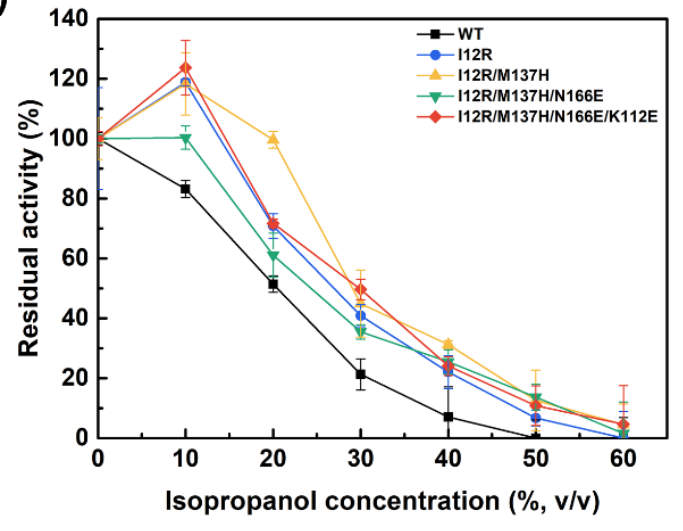

(b)

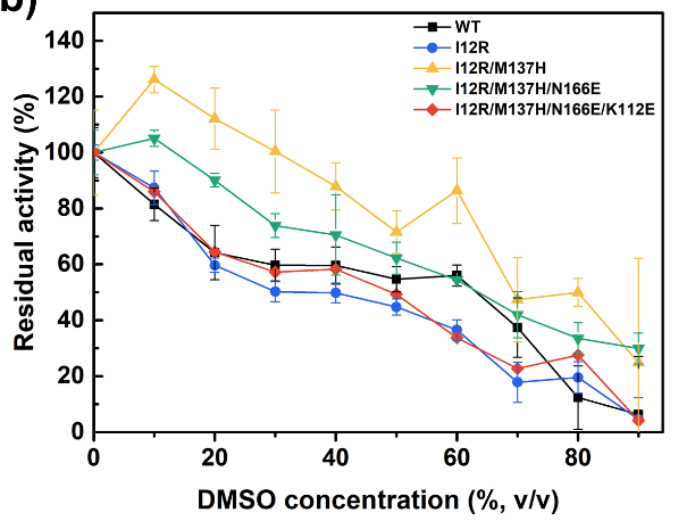

Figure S38. Multiple OS resistance relative to BSLA WT of the purified BSLA recombinants in the varied concentration of OSs (a) isopropanol, (b) DMSO. Resistance was measured after incubating for $2 \mathrm{~h}$ in OSs at room temperature. All data shown are average values from measurements in triplicates or more. 


\section{Reference}

1. Zhang, L.; Cui, H.; Dhoke, G. V.; Zou, Z.; Sauer, D. F.; Davari, M. D.; Schwaneberg, U., Engineering of laccase $\mathrm{CueO}$ for improved electron transfer in bioelectrocatalysis by semi-rational design. Chem. Eur. J 2020, 26 (22), 4974-4979.

2. Sereda, T.; Mant, C.; Hodges, R., Use of sodium perchlorate at low $\mathrm{pH}$ for peptide separations by reversed-phase liquid chromatography: Influence of perchlorate ion on apparent hydrophilicity of positively charged amino acid side-chains. J. Chromatogr. A 1997, 776 (1), 153-165.

3. Broos, J.; Visser, A. J.; Engbersen, J. F.; Verboom, W.; van Hoek, A.; Reinhoudt, D. N., Flexibility of enzymes suspended in organic solvents probed by time-resolved fluorescence anisotropy. Evidence that enzyme activity and enantioselectivity are directly related to enzyme flexibility. J. Am. Chem. Soc. 1995, 117 (51), 12657-12663.

4. Cui, H.; Stadtmüller, T. H. J.; Jiang, Q.; Jaeger, K. E.; Schwaneberg, U.; Davari, M. D., How to engineer organic solvent resistant enzymes: Insights from combined molecular dynamics and directed evolution study. ChemCatChem 2020, 12, 4073.

5. Dutta Banik, S.; Nordblad, M.; Woodley, J. M.; Peters, G. n. H., A correlation between the activity of Candida antarctica lipase B and differences in binding free energies of organic solvent and substrate. ACS Catal. 2016, 6 (10), 6350-6361.

6. Khmelnitsky, Y. L.; Belova, A. B.; Levashov, A. V.; Mozhaev, V. V., Relationship between surface hydrophilicity of a protein and its stability against denaturation by organic solvents. FEBS Lett. 1991, 284 (2), 267-269. 
7. Kabsch, W.; Sander, C., Dictionary of protein secondary structure: pattern recognition of hydrogen-bonded and geometrical features. Biopolymers 1983, 22 (12), 2577-637.

8. Zhao, J.; Jia, N.; Jaeger, K. E.; Bocola, M.; Schwaneberg, U., Ionic liquid activated Bacillus subtilis lipase A variants through cooperative surface substitutions. Biotechnol. Bioeng. 2015, 112 (10), 1997-2004.

9. Eisenhaber, F.; Lijnzaad, P.; Argos, P.; Sander, C.; Scharf, M., The double cubic lattice method: efficient approaches to numerical integration of surface area and volume and to dot surface contouring of molecular assemblies. J. Comput. Chem. 1995, 16 (3), 273-284.

10. van Pouderoyen, G.; Eggert, T.; Jaeger, K. E.; Dijkstra, B. W., The crystal structure of Bacillus subtilis lipase: a minimal $\alpha / \beta$ hydrolase fold enzyme. J. Mol. Biol. 2001, 309 (1), 215226.

11. Guerois, R.; Nielsen, J. E.; Serrano, L., Predicting changes in the stability of proteins and protein complexes: a study of more than 1000 mutations. J. Mol. Biol. 2002, 320 (2), 369-387.

12. Van Durme, J.; Delgado, J.; Stricher, F.; Serrano, L.; Schymkowitz, J.; Rousseau, F., A graphical interface for the FoldX forcefield. Bioinformatics 2011, 27 (12), 1711-1712.

13. Krieger, E.; Koraimann, G.; Vriend, G., Increasing the precision of comparative models with YASARA NOVA—a self-parameterizing force field. Proteins: Struct., Funct., Bioinf. 2002, 47 (3), 393-402.

14. Cui, H.; Cao, H.; Cai, H.; Jaeger, K. E.; Davari, M. D.; Schwaneberg, U., Computerassisted Recombination (CompassR) teaches us how to recombine beneficial substitutions from directed evolution campaigns. Chem. Eur. J 2020, 26 (3), 643-649. 
15. Wedberg, R.; Abildskov, J.; Peters, G. H., Protein dynamics in organic media at varying water activity studied by molecular dynamics simulation. J. Phys. Chem. B 2012, 116 (8), 25752585 .

16. Kabsch, W.; Sander, C., Dictionary of protein secondary structure: pattern recognition of hydrogen-bonded and geometrical features. Biopolymers 1983, 22 (12), 2577-2637. 